\title{
Espaços-tempos assintoticamente planos
}

\author{
Eder Santana Annibale
}

DISSERTAÇÃO APRESENTADA

$\mathrm{AO}$

INSTITUTO DE MATEMÁTICA E ESTATÍSTICA

DA

UNIVERSIDADE DE SÃO PAULO

PARA

OBTENÇÃO DO GRAU DE MESTRE

EM

CIÊNCIAS

Área de Concentração: Matemática Aplicada

Orientador: Prof. Dr. Michael Forger

Durante a elaboração deste trabalho o autor recebeu apoio financeiro do CNPq

- São Paulo, março de 2007 - 



\section{Espaços-tempos assintoticamente planos}

Este exemplar corresponde à redação final da dissertação devidamente corrigida e defendida por Eder Santana Annibale e aprovada pela comissão julgadora.

São Paulo, 02 de março de 2007.

\section{COMISSÃO JULGADORA}

- Prof. Dr. Frank Michael Forger (orientador) - IME-USP

- Prof. Dr. Elcio Abdalla - IF-USP

- Prof. Dr. Alberto Saa - IMECC-UNICAMP 



\title{
Resumo
}

Neste trabalho investigamos a base matemática de uma nova técnica para relacionar duas métricas em uma dada variedade que propomos chamar de "reescalonamento conforme anisotrópico" e que tem sido usada na literatura recente para dar uma nova e mais geométrica definição da noção de espaços-tempos assintoticamente planos em Relatividade Geral.

\begin{abstract}
In this thesis, we investigate the mathematical basis of a new technique for relating two metrics on a given manifold that we propose to call "anisotropic conformal rescaling" and that has been used in the recent literature to give a new and more geometric definition of the notion of asymptotically flat space-times in general relativity.
\end{abstract}





\section{Agradecimentos}

Ao Prof. Michael Forger, meu orientador, pela dedicação, paciência e disponibilidade. Seus conhecimentos e seu profissionalismo contribuíram veementemente na minha formação acadêmica e incentivaram-me a concluir esta dissertação e a dar início a uma nova etapa. A minha família e meus amigos pelo apoio incondicional e ao CNPq por financiar este trabalho durante dois anos. 



\section{Conteúdo}

Introdução

1 Reescalonamento conforme $\quad 7$

1.1 A noção de completamento . . . . . . . . . . . . . . . 7

1.2 Reescalonamento conforme isotrópico . . . . . . . . . . . . . 9

1.3 Reescalonamento conforme anisotrópico . . . . . . . . . . . . . . . . . 10

1.4 Espaços-tempos assintoticamente planos . . . . . . . . . . . . . 25

2 Soluções de Schwarzschild e de Reissner-Nordström 31

2.1 Soluções exatas com simetria esférica . . . . . . . . . . . . . . . . . . . . 31

2.2 Completamento conforme anisotrópico . . . . . . . . . . . . . 35

$\begin{array}{ll}\text { Conclusão } & 41\end{array}$

$\begin{array}{ll}\text { Bibliografia } & 43\end{array}$ 



\section{Introdução}

A presente dissertação trata do problema de apresentar uma definição adequada do conceito de um espaço-tempo assintoticamente plano, tema que desempenha um papel importante na relatividade geral, pois descreve o campo gravitacional gerado por uma distribuição de massa localizada em uma região limitada do espaço. De fato, as fontes gravitacionais usuais da astrofísica, tais como o nosso sistema solar ou como qualquer outro corpo astronômico (por exemplo, uma estrela normal, uma estrela de nêutrons ou mesmo um buraco negro formado por colapso gravitacional), ocupam regiões limitadas do espaço e é razoável imaginar que, dentro e nas proximidades de tais fontes, o espaço-tempo é curvo, enquanto que, à medida que nos afastamos da fonte, a curvatura decresce e a métrica do espaço-tempo aproxima-se da métrica plana do espaço-tempo de Minkowski.

Para caracterizar este tipo de situação de forma tecnicamente correta, precisamos, antes de mais nada, especificar como quantificar o conteúdo da expressão "afastar-se da fonte". O procedimento mais direto, adotado quase universalmente durante muito tempo, inclusive no trabalho famoso de Arnowitt, Deser \& Misner [1] sobre a definição da "massa ADM" (ou mais exatamente, do vetor de energia-momento total) de um espaço-tempo assintoticamente plano consiste em introduzir um sistema de coordenadas contendo uma "variável radial" $r$ e impor condições de decaimento apropriadas sobre as componentes do tensor métrico (ou mais exatamente, sobre as diferenças entre as componentes do tensor métrico e do tensor métrico plano de Minkowski), assim como sobre as suas derivadas, à medida em que $r \rightarrow \infty$. O problema principal com esta definição de um espaço-tempo assintoticamente plano é que ela depende do sistema de coordenadas empregado. Assim, é necessário formular condições geométricas, invariantes sob transformações de coordenadas, que garantam a existência de um tal sistema de coordenadas e de encontrar um algoritmo para construi-lo. A rigor, nem é claro "a priori" qual é o significado geométrico da "variável radial" $r$ e, logo, o do limite $r \rightarrow \infty$. 
Quanto à última questão, podemos tentar uma resposta simples e direta: este limite significa que a distância entre a fonte e o observador tende ao infinito. Contudo, ao contrário de uma métrica riemanniana, onde "distância" significa simplesmente "distância geodésica", essa interpretação do referido limite pode, no caso de uma métrica lorentziana, deixar de ser adequada, uma vez que há pontos cuja distância geodésica é zero, pois podem ser ligados por geodésicas tipo-luz.

Geodésicas em variedades lorentzianas podem ser divididas naturalmente em três tipos: geodésicas tipo-tempo, geodésicas tipo-espaço e geodésicas tipo-luz (ou nulas), pois segue da equação geodésica,

$$
\frac{d^{2} x^{a}}{d \lambda^{2}}+\Gamma_{b c}^{a}(x) \frac{d x^{b}}{d \lambda} \frac{d x^{c}}{d \lambda}=0
$$

a qual fixa inclusive o parâmetro $\lambda$ a menos de uma transformação afim, motivando assim a denominação "parâmetro afim", que o vetor velocidade com respeito a este parâmetro tem comprimento constante ao longo de qualquer geodésica,

$$
\frac{d}{d \lambda}\left(g_{a b}(x) \frac{d x^{a}}{d \lambda} \frac{d x^{b}}{d \lambda}\right)=0
$$

e portanto, uma geodésica cujo vetor tangente inicial é tipo-tempo, tipo-espaço ou tipoluz terá vetor tangente tipo-tempo, tipo-espaço ou tipo-luz ao longo de toda a trajetória. Quando lidamos com curvas tipo-tempo ou tipo-espaço, a equação (2) pode ser usada como definição alternativa do conceito de "parâmetro afim", sendo que o parâmetro afim natural para curvas tipo-tempo é o tempo próprio $\tau$ e para curvas tipo-espaço é o comprimento de arco $l$. Vale observar que estes parâmetros podem ser definidos para curvas arbitrárias (geodésicas ou não), a partir de um parâmetro arbitrário $\lambda$, através das equações ${ }^{1}$

$$
\begin{gathered}
\tau(\lambda)=\frac{1}{c} \int^{\lambda} d \lambda^{\prime} \sqrt{-g_{a b}(x) \frac{d x^{a}}{d \lambda^{\prime}} \frac{d x^{b}}{d \lambda^{\prime}}}, \\
l(\lambda)=\int^{\lambda} d \lambda^{\prime} \sqrt{g_{a b}(x) \frac{d x^{a}}{d \lambda^{\prime}} \frac{d x^{b}}{d \lambda^{\prime}}} .
\end{gathered}
$$

Assim, torna-se possível definir de forma precisa o que, em uma variedade lorentziana (orientada no tempo), significa "ir ao infinito" ao longo de direções temporais (para o passado ou para o futuro) e ao longo de direções espaciais, mas não ao longo de direções nulas (para o passado ou para o futuro). Em particular, a noção de distância geodésica é inadequada para estudar o comportamento assintótico (condições de decaimento) de radiação - seja eletromagnética, seja gravitacional.

\footnotetext{
${ }^{1}$ Seguimos a convenção usual em Relatividade Geral, segundo a qual o tensor métrico tem assinatura $(-+++)$.
} 
Um método alternativo para abordar o problema, originalmente proposto por Penrose [2], consiste em acrescentar ao espaço-tempo uma fronteira representando o "infinito", o que permite formular, de maneira manifestamente independente da escolha de coordenadas específicas, condições sobre o comportamento assintótico do campo gravitacional, assim como de todos os demais campos, em termos de continuidade ou até mesmo de diferenciabilidade perto da fronteira. Mais especificamente, Penrose usou a técnica do "completamento conforme", baseada na introdução de uma métrica auxiliar, não-física, mas conformemente equivalente à métrica física, que permite uma extensão natural a uma fronteira que consiste de duas componentes conexas, o "infinito nulo futuro" $\mathrm{J}^{+}$e o "infinito nulo passado" $\mathrm{J}^{-}$.

Esta construção é descrita em vários livros texto da área [3, 4], mas como já pode ser visto no caso do "completamento conforme" do espaço-tempo plano de Minkowski, apresenta um defeito sério: descreve bem o "infinito nulo" (futuro e passado), mas não o "infinito temporal" (futuro e passado) e, o que é pior, nem o "infinito espacial", que são representados, cada um, por apenas um ponto, denotados por $i^{+}, i^{-}$e $i^{0}$, respectivamente. Este defeito é compartilhado nas abordagens de Geroch [5] e de Ashtekar \& Hansen [6], que também usam o método de "completamento conforme" para chegar, respectivamente, a uma descrição do infinito espacial e de uma unificação do infinito nulo e espacial: em ambos os casos, o infinito espacial é comprimido em um único ponto, fato que é reponsável por grande parte das dificuldades inerentes destas abordagens.

Uma análise mais aprofundada das origens do problema revela que se trata de uma dificuldade fundamental, cuja dimensão e extensão parece ter sido subestimada durante décadas. Na verdade, como observado por Persides em [7], ela aparece não apenas no âmbito da geometria lorentziana mas, da mesma forma, no da geometria riemanniana, onde já pode ser apreciada considerando-se o exemplo mais simples possível: a questão de descrever o "infinito" para o espaço euclideano $\mathbb{R}^{3}$.

A fim de preservar a simetria rotacional, consideremos o espaço euclideano com a origem removida, $M=\mathbb{R}^{3} \backslash\{0\}$, em coordenadas esféricas $(r, \theta, \varphi)$ :

$$
\begin{gathered}
x=r \sin \theta \cos \varphi \\
y=r \sin \theta \sin \varphi \\
z=r \cos \theta
\end{gathered}
$$

Nestas coordenadas a métrica do espaço euclideano assume a forma

$$
d s^{2}=d r^{2}+r^{2}\left(d \theta^{2}+\sin ^{2} \theta d \varphi^{2}\right) .
$$

É natural considerar o "infinito" como sendo a fronteira de um completamento ${ }^{2} \bar{M}$ de $M$, obtida tomando-se o limite $r \rightarrow \infty$, $\operatorname{com} \theta$ e $\varphi$ constantes. Para formalizar esta idéia,

\footnotetext{
${ }^{2}$ Uma definição rigorosa desta noção será apresentada no próximo capítulo.
} 
introduzimos uma nova coordenada radial, $\Omega=1 / r$, e estendemos $M$ de forma a incluir os pontos onde $\Omega$ se anula. Nas novas coordenadas esféricas $(\Omega, \theta, \varphi)$, a métrica do espaço euclideano assume a forma

$$
d s^{2}=\Omega^{-4} d \Omega^{2}+\Omega^{-2}\left(d \theta^{2}+\sin ^{2} \theta d \varphi^{2}\right) .
$$

Obviamente, esta métrica diverge no limite $\Omega \rightarrow 0$, sendo que os pontos de $M$ com coordenada radial $\Omega$ formam uma esfera $S^{2}$ com área total $4 \pi / \Omega^{2}$, a qual tende para $\infty$ quando $\Omega \rightarrow 0$. Contudo, se efetuarmos um "reescalonamento conforme"

$$
d s^{2} \longrightarrow d \tilde{s}^{2}=\Omega^{4} d s^{2},
$$

a nova métrica

$$
d \tilde{s}^{2}=d \Omega^{2}+\Omega^{2}\left(d \theta^{2}+\sin ^{2} \theta d \varphi^{2}\right)
$$

converge no limite $\Omega \rightarrow 0$ e portanto pode ser estendida a uma métrica regular em $\bar{M}$. O problema com este procedimento é que, nesta nova métrica, os pontos de $M$ com coordenada radial $\Omega$ formam uma esfera $S^{2}$ com área total $4 \pi \Omega^{2}$, a qual tende para 0 quando $\Omega \rightarrow 0$. Isso significa que a fronteira de $\bar{M}$ tem área total 0 e portanto, se reduz a um único ponto: estamos lidando com a compactificação de um ponto, ao invés de uma compactificação que represente o "infinito" por uma esfera $S^{2}$. Podemos dizer que o simples reescalonamento conforme provoca um "encolhimento exagerado" das esferas no limite $\Omega \rightarrow 0$.

A solução do problema, neste caso, é óbvia: consiste em abandonar o preconceito de que a nova métrica deva ser obtida a partir da métrica original por um simples reescalonamento conforme e trabalhar com a métrica

$$
d \hat{s}^{2}=d \Omega^{2}+\left(d \theta^{2}+\sin ^{2} \theta d \varphi^{2}\right)
$$

que também converge no limite $\Omega \rightarrow 0$ e portanto pode ser estendida a uma métrica regular em $\bar{M}$, mas com o mérito adicional que agora, os pontos de $M$ com coordenada radial $\Omega$ formam uma esfera $S^{2}$ com área total $4 \pi$, a qual tende a um valor finito e nãonulo $(4 \pi$, no caso) quando $\Omega \rightarrow 0$. O preço a pagar é que a relação entre a nova métrica e a métrica original é mais complicada, sendo que $d \hat{s}^{2}$ é obtida de $d s^{2}$ reescalonandose as suas componentes tangenciais e normais às superfícies $\Omega=$ const. por potências diferentes de $\Omega$ : escrevendo

$$
d s^{2}=d s_{\perp}^{2}+d s_{\|}^{2} \quad, \quad d \hat{s}^{2}=d \hat{s}_{\perp}^{2}+d \hat{s}_{\|}^{2},
$$

temos

$$
\begin{aligned}
d \hat{s}_{\perp}^{2} & =\Omega^{4} d s_{\perp}^{2} \\
d \hat{s}_{\|} & =\Omega^{2} d s_{\|}^{2}
\end{aligned}
$$


É por isso que nos referiremos à passagem

$$
d s^{2} \longrightarrow d \hat{s}^{2}
$$

como um "reescalonamento conforme anisotrópico".

Como mostra este exemplo, uma descrição geométrica do "infinito espacial", através de um completamento que leve a uma variedade com bordo, cujo interior é igual à variedade original, mas munida de uma métrica diferente da original, requer o reescalonamento das componentes tangenciais e transversais da métrica original por potências diferentes da "variável radial" $\Omega$ e portanto, não pode ser baseada na idéia do completamento conforme. Este problema só não aparece no caso lorentziano quando a direção transversal à fronteira é tipo-luz, e é por isso que o completamento conforme permite tratar o "infinito nulo" mas não o "infinito espacial" ou "infinito temporal".

A inadequação do completamento conforme usual para tratar do "infinito espacial" já foi percebida nos anos 70 e exposta em vários trabalhos, levando inclusive a tentativas de abordagens alternativas tais como o completamento projetivo proposto em [8] e as expansões da métrica elaboradas em [9]. O passo decisivo, no entanto, só foi tomado nos anos 90, com o trabalho pioneiro de Ashtekar e Romano [10] e as melhorias introduzidas por Perng [11].

Em [10] é apresentada uma definição geométrica do conceito de um espaço-tempo assintoticamente plano no infinito espacial que realiza o "infinito espacial" com o devido grau de regularidade, a saber, como uma variedade tridimensional constituindo o bordo do espaço-tempo em suas direções espaciais (algumas ou todas elas). Os autores também deduzem uma série de conseqüências de sua definição, inclusive a construção de quantidades conservadas associadas a simetrias assintóticas, tais como energia-momento e momento angular, a partir de expressões para certos campos tensoriais que descrevem, em primeira ordem, o comportamento assintótico do campo gravitacional no infinito espacial. Em [11], a definição de [10] é ligeiramente modificada, de modo a incluir o importante caso da solução de Reissner-Nordström, e a expansão assintótica de campos físicos é sistematizada e levada a ordens superiores, o que permite deduzir um conjunto completo de possíveis quantidades conservadas.

O objetivo desta dissertação é apresentar uma introdução sistemática e completa aos passos iniciais da metodologia adotada em [10, 11], que propomos chamar de "reescalonamento conforme anisotrópico" e que tem o procedimento descrito acima como o seu exemplo mais elementar. Em particular, enfatizamos a importância da "métrica nãofísica", que constitui uma ferramenta útil para unificar e melhor compreender algumas das condições (aparentemente independentes) impostas na definição original de [10, 11]. De fato, como já acontece no caso da definição do "infinito nulo" por completamento conforme, devido a Penrose, a condição de que a métrica não-física, construída a partir 
da métrica física segundo uma receita específica, admita uma extensão suave (i.e., não apenas contínua) ao bordo impõe todo um conjunto de restrições sobre a métrica física original e todas as suas derivadas. A mesma condição é imposta sobre todos os outros campos tensoriais físicos, desde que devidamente reescalonados. Esta condição universal será a única condição de continuidade/diferenciabilidade a ser exigida em todo o formalismo, substituindo todas as outras e assim eliminando a necessidade de introduzir condições de diferenciabilidade estranhas ou "limites com dependência direcional" para descrever o comportamento de campos físicos no bordo, o que é inevitável quando se representa o "infinito espacial" por um único ponto $i^{0}$, como no caso do completamento conforme. (Veja, por exemplo, [4, pp. 276-277].)

Este trabalho está dividido em dois capítulos principais. No primeiro capítulo apresentamos a definição de completamento de uma variedade, a qual é fundamental para definirmos o infinito espacial como sendo o bordo de um espaço-tempo. Em seguida desenvolvemos o conceito de reescalonamento conforme anisotrópico, método que generaliza o reescalonamento conforme usual (que chamaremos de "isotrópico") e, como este, relaciona duas métricas: uma delas é a "métrica física" que define a geometria do espaço-tempo mas cujas componentes podem divergir no bordo da variedade, enquanto que a outra, que chamaremos de "métrica não-física", é um tensor métrico auxiliar que, em geral, não terá nenhum significado físico mas apresenta a vantagem de que as suas componentes permanecem finitas e regulares (i.e., suaves) no bordo da variedade. ${ }^{3}$ Este método permite estabelecer relações entre as componentes de vários outros "campos físicos", entre eles o tensor de Einstein derivado da métrica física, que novamente podem divergir no bordo, e as componentes dos correspondentes "campos não-físicos", entre eles o tensor de Einstein derivado da métrica não-física, que por construção permanecem finitas e regulares (i.e., suaves) no bordo. Usando tais relações, apresentamos a definição de um espaço-tempo assintoticamente plano no infinito espacial. Finalmente, analisamos algumas conseqüências desta definição e comparamos-na com as definições propostas em $[10,11]$. No segundo capítulo, apresentamos as características básicas das soluções de Schwarzschild e de Reissner-Nordström e aplicamos o formalismo desenvolvido no primeiro capítulo a estas soluções para concluir que a nossa definição engloba estes dois importantes exemplos. Como conclusão do trabalho, mencionamos algumas questões adicionais e problemas em aberto que poderiam e deveriam ser tratados no âmbito da abordagem aqui proposta.

\footnotetext{
${ }^{3}$ Lembramos que segundo os princípios da Relatividade Geral, a métrica se destaca dentre os diversos tipos de campos que existem no espaço-tempo pelo fato de que ela codifica a sua geometria - em particular, a sua estrutura causal (veja, por exemplo, a discussão em [3, Capítulo 3.2, pp. 59-64]), sem permitir a existência de algum tipo de "geometria a priori" (veja, por exemplo, a discussão em [12, $\S 17.6$, pp. 429-431]. Não existe portanto a possibilidade de atribuir igual significado físico a dois tensores métricos do mesmo tempo: se um é físico, o outro não é, ainda mais quando os dois não são conformemente equivalentes e portanto definem estruturas causais diferentes.
} 
CAPÍtulo 1

\section{Reescalonamento conforme isotrópico e anisotrópico}

\subsection{A noção de completamento}

Começamos por um resumo daqueles aspectos da noção de "completamento" que independem da natureza específica da relação entre as duas métricas envolvidas - a métrica "física" $g$ e a métrica "não-física" $\bar{g}$.

Definição 1.1 Um completamento de uma variedade $M$ é um $\operatorname{par}(\bar{M}, \Omega)$, onde $\bar{M}$ é uma variedade com bordo $\partial \bar{M}$ tal que a variedade $M$ pode (através de um difeomorfismo escolhido de uma vez por todas) ser identificada com o interior $\bar{M} \backslash \partial \bar{M}$ de $\bar{M}$ e $\Omega$ é uma função sobre $\bar{M}$ que serve como coordenada transversal ao bordo de $\bar{M}$, isto é, que satisfaz

$$
\Omega>0 \text { sobre } M \quad, \quad \Omega=0 \text { sobre } \partial \bar{M},
$$

e

$$
d \Omega \neq 0 \text { sobre } \partial \bar{M} \text {. }
$$

Mais especificamente, exigimos a existência de uma vizinhança colar ${ }^{1}$ de $\partial \bar{M}$ em $\bar{M}$, isto é, de uma vizinhança aberta de $\partial \bar{M}$ em $\bar{M}$ da forma

$$
\bar{U}_{\epsilon}=\{x \in \bar{M} \mid \Omega(x)<\epsilon\},
$$

com $\epsilon>0$, tal que

$$
d \Omega \neq 0 \text { sobre } \bar{U}_{\epsilon} .
$$

\footnotetext{
${ }^{1}$ No uso deste termo, seguimos [13].
} 
É claro que, nestas hipóteses, as superfícies de nível de $\Omega$

$$
S_{\rho}=\{x \in \bar{M} \mid \Omega(x)=\rho\},
$$

com $0 \leqslant \rho<\epsilon$, são subvariedades de $\bar{M}$ (e, para $\rho>0$, de $M$, enquanto que $S_{0}=\partial \bar{M}$ ) que providenciam uma folheação da vizinhança colar $\bar{U}_{\epsilon}$ :

$$
\bar{U}_{\epsilon}=\bigcup_{0 \leqslant \rho<\epsilon} S_{\rho}
$$

Escrevemos, ainda,

$$
U_{\epsilon}=\bigcup_{0<\rho<\epsilon}^{\cdot} S_{\rho} .
$$

Assim, $\bar{U}_{\epsilon}$ é a união disjunta de $\partial \bar{M}$ e $U_{\epsilon}$ :

$$
\bar{U}_{\epsilon}=\partial \bar{M} \dot{\cup} U_{\epsilon}
$$

Também notamos que a existência de uma vizinhança colar de $\partial \bar{M}$, que é uma vizinhança aberta de $\partial \bar{M}$ de "espessura constante ao longo de $\partial \bar{M}$ " ( $\epsilon$, no caso) é automaticamente garantida pelas hipóteses anteriores se $\partial \bar{M}$ for compacto.

Passando a incluir métricas, adotaremos a seguinte terminologia.

Definição 1.2 Um completamento de uma variedade pseudo-riemanniana $(M, g)$ é uma tripla $(\bar{M}, \bar{g}, \Omega)$, onde $(\bar{M}, \bar{g})$ é uma variedade pseudo-riemanniana do mesmo tipo, mas com bordo $\partial \bar{M}$, e $(\bar{M}, \Omega)$ é um completamento de $M$ como na definição anterior, tal que, sobre cada uma das superfícies de nível $S_{\rho}$ de $\Omega$, com $0<\rho<\epsilon$, as métricas sobre $S_{\rho}$ induzidas por $g,\left.g\right|_{S_{\rho}}$, e por $\bar{g},\left.\bar{g}\right|_{S_{\rho}}$, assim como os campos vetoriais normais unitários $n$ para $g$ e $\bar{n}$ para $\bar{g}$, são proporcionais, sendo que, para garantir que na região $U_{\epsilon}, \bar{g}$ seja completamente determinado por $g$ e $\Omega$ e, reciprocamente, que g também seja completamente determinado por $\bar{g}$ e $\Omega$, os respectivos fatores de proporcionalidade podem ser escritos como funções de $\Omega$ (e portanto são constantes ao longo de cada uma das superfícies de nível $S_{\rho}$ de $\Omega$, com $0<\rho<\epsilon$ ).

Nas aplicações em relatividade geral, $g$ é interpretado com o tensor métrico "físico" que, tipicamente, diverge à medida em que nos aproximamos do bordo $\partial \bar{M}$ de $\bar{M}$, enquanto que $\bar{g}$ é um tensor métrico sem significado físico que, porém, permanece regular mesmo no bordo $\partial \bar{M}$ de $\bar{M}$. 


\subsection{Reescalonamento conforme isotrópico}

Dada uma variedade pseudo-riemanniana qualquer, a maneira mais simples de acrescentar uma fronteira representando pontos "no infinito" é através de um "completamento conforme", isto é, supondo que a métrica não-física (que neste caso denotaremos por $\tilde{g}$ ) é obtida a partir da métrica física $g$ por um simples reescalonamento conforme.

Seja $M$ uma variedade munida de uma métrica pseudo-riemanniana $g$. No que segue, estaremos interessados em dois casos distintos: métricas riemannianas e métricas lorentzianas. Dada uma função $\Omega$ estritamente positiva sobre $M$, que escrevemos na forma $\Omega=\exp (\omega)$, podemos introduzir uma nova métrica $\tilde{g}$ sobre $M$ através de

$$
\tilde{g}=\exp (2 \omega) g .
$$

Dizemos que a métrica $\tilde{g}$ é obtida da métrica $g$ por um reescalonamento conforme ou, mais precisamente, por um reescalonamento conforme isotrópico, para distingui-lo do reescalonamento conforme anisotrópico que será introduzido mais tarde. Entende-se que a fórmula (1.9) aplica-se aos tensores covariantes, enquanto que para os tensores contravariantes vale uma relação análoga com a potência inversa de $\Omega$, ou seja, temos em componentes:

$$
\tilde{g}_{a b}=\exp (2 \omega) g_{a b} \quad, \quad \tilde{g}^{a b}=\exp (-2 \omega) g^{a b} .
$$

Quanto aos símbolos de Christoffel das respectivas conexões de Levi-Civita, observamos que estes são invariantes por reescalonamentos conformes globais ( $\omega$ é constante), enquanto que no caso de reescalonamentos locais ( $\omega$ é uma função), obtemos a relação

$$
\tilde{\Gamma}_{a b}^{c}=\Gamma_{a b}^{c}+\left(\delta_{a}^{c} \partial_{b} \omega+\delta_{b}^{c} \partial_{a} \omega-g_{a b} g^{c d} \partial_{d} \omega\right) .
$$

Logo, valem as seguintes relações entre os respectivos tensores de Riemann

$$
\begin{aligned}
\tilde{R}_{b c d}^{a}= & R_{b c d}^{a}-\left(\delta_{c}^{a} \nabla_{d} \partial_{b} \omega-\delta_{d}^{a} \nabla_{c} \partial_{b} \omega\right)+\left(g_{b c} g^{a e} \nabla_{d} \partial_{e} \omega-g_{b d} g^{a e} \nabla_{c} \partial_{e} \omega\right) \\
& +\left(\delta_{c}^{a} \partial_{d} \omega \partial_{b} \omega-\delta_{d}^{a} \partial_{c} \omega \partial_{b} \omega\right)-\left(g_{b c} g^{a e} \partial_{d} \omega \partial_{e} \omega-g_{b d} g^{a e} \partial_{c} \omega \partial_{e} \omega\right) \\
& -\left(\delta_{c}^{a} g_{d b}-\delta_{d}^{a} g_{c b}\right)(\partial \omega)^{2},
\end{aligned}
$$

entre os respectivos tensores de Ricci

$$
\tilde{R}_{a b}=R_{a b}-(n-2) \nabla_{b} \partial_{a} \omega-g_{a b} \partial^{2} \omega+(n-2) \partial_{a} \omega \partial_{b} \omega-(n-2) g_{a b}(\partial \omega)^{2}
$$

e entre as respectivas curvaturas escalares

$$
\tilde{R}=\exp (-2 \omega)\left(R-2(n-1) \partial^{2} \omega-(n-1)(n-2)(\partial \omega)^{2}\right),
$$

sendo o tensor de Weyl invariante por reescalonamentos conformes; veja, por exemplo, [3, Chapter 2.6, p. 42] ou [14], eqs (295)-(298), p. 384. 
Também vale observar que, no caso lorentziano, reescalonamentos conformes preservam geodésicas nulas, a menos de uma reparametrização. De fato, seja $x$ uma geodésica para a métrica $g$ com parâmetro afim $\lambda$. Então, se introduzirmos um novo parâmetro $\tilde{\lambda}$ dado por simples integração da relação

$$
\frac{d \tilde{\lambda}}{d \lambda}=\exp (2 \omega(x))
$$

a mesma curva considerada como função de $\tilde{\lambda}$ será uma geodésica para a métrica $\tilde{g}$ com parâmetro afim $\tilde{\lambda}$, desde que

$$
g_{a b}(x) \frac{d x^{a}}{d \lambda} \frac{d x^{b}}{d \lambda}=0
$$

pois

$$
\frac{d x^{a}}{d \tilde{\lambda}}=\exp (-2 \omega(x)) \frac{d x^{a}}{d \lambda}
$$

$\mathrm{e}$

$$
\frac{d^{2} x^{a}}{d \tilde{\lambda}^{2}}=\exp (-4 \omega(x))\left(\frac{d^{2} x^{a}}{d \lambda^{2}}-2\left(\partial_{c} \omega\right)(x) \frac{d x^{c}}{d \lambda} \frac{d x^{a}}{d \lambda}\right)
$$

e portanto, conforme a equação (1.11),

$$
\begin{aligned}
\frac{d^{2} x^{a}}{d \tilde{\lambda}^{2}} & +\tilde{\Gamma}_{b c}^{a}(x) \frac{d x^{b}}{d \tilde{\lambda}} \frac{d x^{c}}{d \tilde{\lambda}} \\
& =\exp (-4 \omega(x))\left(\frac{d^{2} x^{a}}{d \lambda^{2}}+\Gamma_{b c}^{a}(x) \frac{d x^{b}}{d \lambda} \frac{d x^{c}}{d \lambda}-\left(g^{a b} \partial_{b} \omega\right)(x) g_{c d}(x) \frac{d x^{c}}{d \lambda} \frac{d x^{d}}{d \lambda}\right) .
\end{aligned}
$$

\subsection{Reescalonamento conforme anisotrópico}

Um outro método de reescalonamento, que nós chamaremos de anisotrópico, usa a decomposição dos espaços tangentes ao espaço-tempo em sua parte tangencial e sua parte normal às hipersuperfícies de nível da própria função de reescalonamento $\Omega$. Para descrevê-lo, considere primeiro uma variedade pseudo-riemanniana $(M, g)$ munida de uma função $\Omega$ tal que $d \Omega \neq 0$ sobre $M$ e, mais especificamente, no caso em que $g$ não for definida positiva, tal que o gradiente $(d \Omega)^{\sharp}$ de $\Omega$ é um campo vetorial tipo-espaço sobre $M$. Sob estas hipóteses, as superfícies de nível de $\Omega$ definem uma folheação de $M$ em hipersuperfícies que são pseudo-riemannianas em relação à métrica $q$ induzida sobre cada uma pela métrica original $g$. Definimos então o campo vetorial unitário $n$ normal a esta folheação por $n^{b}=F^{-1 / 2}(d \Omega)^{\sharp}$, ou seja,

$$
n^{a}=F^{-1 / 2} g^{a b} \partial_{b} \Omega
$$


e a correspondente 1-forma unitária $n^{b}$ por $n^{b}=F^{-1 / 2} d \Omega$, ou seja,

$$
n_{a}=F^{-1 / 2} \partial_{a} \Omega
$$

onde

$$
F=g^{a b} \partial_{a} \Omega \partial_{b} \Omega
$$

de modo que vale

$$
g_{a b} n^{a} n^{b}=1=g^{a b} n_{a} n_{b} .
$$

Assim, podemos decompor a métrica $g$ em duas partes: um tensor simétrico $q$ que descreve a sua parte tangencial e um tensor simétrico proporcional a $d \Omega \otimes d \Omega$, ou $n^{b} \otimes n^{b}$, que descreve a sua parte normal:

$$
g_{a b}=q_{a b}+n_{a} n_{b}, \quad g^{a b}=q^{a b}+n^{a} n^{b} .
$$

Vale então

$$
q_{a b} n^{b}=0, q^{a b} n_{b}=0, q_{a c} q^{c b}=P_{a}^{b},
$$

onde $P$ é o projetor ortogonal de $T M$ sobre $\operatorname{ker}(d \Omega)$ :

$$
P_{a}^{b}=\delta_{a}^{b}-n_{a} n^{b}
$$

Valem ainda as seguintes relações:

$$
P_{a}^{b} P_{b}^{c}=P_{a}^{c}, \quad P_{a}^{b} n_{b}=P_{a}^{b} n^{a}=0 .
$$

O tensor simétrico $q$, também chamado de primeira forma fundamental, descreve a métrica induzida sobre cada uma das superfícies de nível de $\Omega$. Outro tensor simétrico muito importante, aqui denotado por $K$, é a segunda forma fundamental, que descreve a curvatura extrínseca das superfícies de nível de $\Omega$ como subvariedades de $M$ e que pode ser definida de várias formas, por exemplo como a derivada covariante do campo normal $n$ (mais exatamente, da 1-forma normal $n^{b}$ ) em direções tangenciais,

$$
K_{a b}=P_{a}^{c} P_{b}^{d} \nabla_{c} n_{d},
$$

que, no presente caso, é simplesmente a parte tangencial das segundas derivadas (covariantes) da função $\Omega$,

$$
K_{a b}=F^{-1 / 2} P_{a}^{c} P_{b}^{d} \nabla_{c} \partial_{d} \Omega
$$

ou como a derivada de Lie da métrica induzida ao longo do campo unitário normal,

$$
K_{a b}=\frac{1}{2} \mathcal{L}_{n} q_{a b}
$$


Observe que $K$ é simétrico, $K_{b a}=K_{a b}$, como pode ser visto, por exemplo, calculando-se, para quaisquer dois campos vetoriais $X$ e $Y$ com projeções tangenciais $P X$ e $P Y$,

$$
\begin{aligned}
& K(X, Y)-K(Y, X)=g\left(\nabla_{P X} n, P Y\right)-g\left(P X, \nabla_{P Y} n\right) \\
& \quad=\mathcal{L}_{P X} g(n, P Y)-g\left(n, \nabla_{P X} P Y\right)-\mathcal{L}_{P Y} g(P X, n)+g\left(\nabla_{P Y} P X, n\right) \\
& \quad=-g(n,[P X, P Y])=0 .
\end{aligned}
$$

A mesma afirmação também segue da equação (1.28), usando que a torção de $\nabla$ é zero, e é óbvia a partir da equação (1.29).

\subsubsection{Equações de Gauss-Codazzi}

Para os cálculos a serem efetuados nesta seção, precisaremos de uma série de fórmulas que relacionam diversas quantidades que aparecem naturalmente quando consideramos uma folheação de uma variedade lorentziana pelas hipersuperfícies de nível de uma função, dentre elas as bem conhecidas equações de Gauss-Codazzi. Mantendo a notação já introduzida, observamos primeiro que combinando as equações

$$
n^{b} \nabla_{a} n_{b}=g^{b c} n_{c} \nabla_{a} n_{b}=n_{c} \nabla_{a} n^{c}
$$

e

$$
n^{b} \nabla_{a} n_{b}+\nabla_{a} n^{b} n_{b}=\partial_{a}\left(n^{b} n_{b}\right)=0
$$

obtemos as seguintes importantes identidades:

$$
n^{b} \nabla_{a} n_{b}=0, n_{b} \nabla_{a} n^{b}=0
$$

Logo, vemos como deduzir a equivalência entre as equações (1.27) e (1.29):

$$
\begin{aligned}
\mathcal{L}_{n} q_{a b} & =n^{c} \nabla_{c} q_{a b}+q_{a c} \nabla_{b} n^{c}+q_{c b} \nabla_{a} n^{c} \\
& =-n^{c} \nabla_{c}\left(n_{a} n_{b}\right)+\left(g_{a c}-n_{a} n_{c}\right) \nabla_{b} n^{c}+\left(g_{c b}-n_{c} n_{b}\right) \nabla_{a} n^{c} \\
& =\nabla_{a} n_{b}-n_{a} n^{c} \nabla_{c} n_{b}+\nabla_{b} n_{a}-n^{c} \nabla_{c} n_{a} n_{b} \\
& =\left(\delta_{a}^{c}-n_{a} n^{c}\right)\left(\delta_{b}^{d}-n_{b} n^{d}\right) \nabla_{c} n_{d}-\left(\delta_{b}^{c}-n_{b} n^{c}\right)\left(\delta_{a}^{d}-n_{a} n^{d}\right) \nabla_{c} n_{d} \\
& =K_{a b}+K_{b a} .
\end{aligned}
$$

Note também que devido à relação (1.30), a divergência do campo vetorial unitário $n$ é igual ao traço $K=g^{a b} K_{a b}=q^{a b} K_{a b}$ da segunda forma fundamental:

$$
\nabla_{a} n^{a}=K
$$


Por outro lado, as definições da métrica induzida $q$ e do projetor $P$ implicam que

$$
\nabla_{a} q_{b c}=-\left(\nabla_{a} n_{b}\right) n_{c}-\left(\nabla_{a} n_{c}\right) n_{b}
$$

$\mathrm{e}$

$$
\nabla_{a} P_{b}^{c}=-\left(\nabla_{a} n_{b}\right) n^{c}-n_{b}\left(\nabla_{a} n^{c}\right)
$$

Quanto às derivadas da função $F$, a derivada normal é dada por

$$
\mathcal{L}_{n} F=n^{a} \partial_{a}\left(g^{c d} \partial_{c} \Omega \partial_{d} \Omega\right)=n^{a} g^{c d}\left(\left(\nabla_{a} \partial_{c} \Omega\right) \partial_{d} \Omega+\partial_{c} \Omega\left(\nabla_{a} \partial_{d} \Omega\right)\right)
$$

ou seja,

$$
\mathcal{L}_{n} F=2 F^{1 / 2} n^{c} n^{d} \nabla_{c} \partial_{d} \Omega,
$$

enquanto que para as derivadas tangenciais, temos

$$
\begin{aligned}
P_{a}^{b} \partial_{b} F & =P_{a}^{b} \partial_{b}\left(g^{c d} \partial_{c} \Omega \partial_{d} \Omega\right)=P_{a}^{b} g^{c d}\left(\left(\nabla_{b} \partial_{c} \Omega\right) \partial_{d} \Omega+\partial_{c} \Omega\left(\nabla_{b} \partial_{d} \Omega\right)\right) \\
& =2 F^{1 / 2} P_{a}^{b} n^{c} \nabla_{b} \partial_{c} \Omega=2 F^{1 / 2} P_{a}^{b} n^{c} \nabla_{c} \partial_{b} \Omega=2 F^{1 / 2} P_{a}^{b} n^{c} \nabla_{c}\left(F^{1 / 2} n_{b}\right) \\
& =2 F P_{a}^{b} n^{c} \nabla_{c} n_{b}=2 F\left(\delta_{a}^{b}-n_{a} n^{b}\right) n^{c} \nabla_{c} n_{b}=2 F n^{c} \nabla_{c} n_{a},
\end{aligned}
$$

ou seja,

$$
P_{a}^{b} \partial_{b} F=2 F^{1 / 2} P_{a}^{b} n^{c} \nabla_{c} \partial_{b} \Omega=2 F P_{a}^{b} n^{c} \nabla_{c} n_{b}=2 F n^{c} \nabla_{c} n_{a}
$$

Reciprocamente, temos

$$
\nabla_{a} n_{b}=\left(P_{a}^{c}+n_{a} n^{c}\right) \nabla_{c} n_{b}=P_{a}^{c}\left(P_{b}^{d}+n_{b} n^{d}\right) \nabla_{c} n_{d}+n_{a} n^{c} \nabla_{c} n_{b}
$$

e portanto

$$
\nabla_{a} n_{b}=K_{a b}+n_{a} n^{c} \nabla_{c} n_{b}=K_{a b}+\frac{1}{2} F^{-1} \partial_{c} F P_{b}^{c} n_{a} .
$$

Finalmente, consideraremos ainda a derivada de Lie da segunda forma fundamental ao longo do campo unitário normal, relacionada com a sua derivada covariante normal pela fórmula

$$
\mathcal{L}_{n} K_{a b}=n^{c} \nabla_{c} K_{a b}+K_{a c} \nabla_{b} n^{c}+K_{c b} \nabla_{a} n^{c},
$$

a qual implica

$$
\begin{aligned}
& n^{a} \mathcal{L}_{n} K_{a b}=n^{a} n^{c} \nabla_{c} K_{a b}+K_{a b} n^{c} \nabla_{c} n^{a}=n^{c} \nabla_{c}\left(n^{a} K_{a b}\right)=0, \\
& n^{b} \mathcal{L}_{n} K_{a b}=n^{b} n^{c} \nabla_{c} K_{a b}+K_{a b} n^{c} \nabla_{c} n^{b}=n^{c} \nabla_{c}\left(n^{b} K_{a b}\right)=0,
\end{aligned}
$$


mostrando que $\mathcal{L}_{n} K_{a b}$, como $q_{a b}$ e $K_{a b}=\frac{1}{2} \mathcal{L}_{n} q_{a b}$, é um tensor puramente tangencial. Reciprocamente, temos

$$
\begin{aligned}
\mathcal{L}_{n} K_{a b} & =n^{c} \nabla_{c} K_{a b}+K_{a c} \nabla_{b} n^{c}+K_{c b} \nabla_{a} n^{c} \\
& =n^{c} \nabla_{c} K_{a b}+\left(q^{c d}+n^{c} n^{d}\right) K_{a c} \nabla_{b} n_{d}+\left(q^{c d}+n^{c} n^{d}\right) K_{c b} \nabla_{a} n_{d} \\
& =n^{c} \nabla_{c} K_{a b}+q^{c d} K_{a c}\left(P_{b}^{e}+n_{b} n^{e}\right) \nabla_{e} n_{d}+q^{c d} K_{c b}\left(P_{a}^{e}+n_{a} n^{e}\right) \nabla_{e} n_{d} \\
& =n^{c} \nabla_{c} K_{a b}+2 q^{c d} K_{a c} K_{b d}+q^{c d}\left(K_{a c} n_{b}+K_{c b} n_{a}\right) n^{e} \nabla_{e} n_{d},
\end{aligned}
$$

o que, em combinação com a equação (1.35), leva à seguinte decomposição da derivada covariante normal da segunda forma fundamental em uma parte puramente tangencial e uma parte mista (sendo que a parte puramente normal se anula):

$$
n^{c} \nabla_{c} K_{a b}=\mathcal{L}_{n} K_{a b}-2 q^{c d} K_{a c} K_{b d}-\frac{1}{2} q^{c d} F^{-1} \partial_{c} F\left(K_{d a} n_{b}+K_{d b} n_{a}\right) .
$$

Passamos agora a deduzir algumas relações entre objetos quadridimensionais e tridimensionais. Em cada superfície de nível de $\Omega$ temos uma derivada covariante tridimensional, que denotaremos por $\mathcal{D}_{a}$, os correspondentes tensores de Riemann e de Ricci tridimensionais, que denotaremos por $\mathcal{R}_{b c d}^{a}$ e $\mathcal{R}_{a b}$, respectivamente, e a correspondente curvatura escalar tridimensional, que denotaremos por $\mathcal{R}$. Primeiro, notamos que o operador $\mathcal{D}$ é definido pela exigência de que para quaisquer dois campos vetoriais $Z$ e $X$ tangentes às superfícies de nível de $\Omega, \mathcal{D}_{Z} X$ será a projeção tangencial de $\nabla_{Z} X$, ou seja, para campos vetoriais $X$ quaisquer, vale

$$
\mathcal{D}_{a} X^{b}=P_{a}^{c} P_{d}^{b} \nabla_{c} X^{d},
$$

e dualmente, para 1-formas $\alpha$ quaisquer, vale

$$
\mathcal{D}_{a} \alpha_{b}=P_{a}^{c} P_{b}^{d} \nabla_{c} \alpha_{d}
$$

Então

$$
\mathcal{R}_{d a b}^{c} X^{d}=\mathcal{D}_{a} \mathcal{D}_{b} X^{c}-\mathcal{D}_{b} \mathcal{D}_{a} X^{c}
$$

e dualmente

$$
\mathcal{R}^{c}{ }_{d a b} \alpha_{c}=\mathcal{D}_{b} \mathcal{D}_{a} \alpha_{d}-\mathcal{D}_{a} \mathcal{D}_{b} \alpha_{d}
$$

Provaremos agora, como exercício, as equações de Gauss-Codazzi,

$$
\begin{aligned}
\mathcal{R}_{d a b}^{c}= & P_{g}^{c} P_{d}^{h} P_{a}^{e} P_{b}^{f} R_{h e f}^{g}+K_{a}^{c} K_{b d}-K_{b}^{c} K_{a d} . \\
& \mathcal{D}_{b} K_{a}^{b}-\mathcal{D}_{a} K=P_{a}^{b} n^{c} R_{b c} .
\end{aligned}
$$


De fato, se $X$ é tangencial às superfícies de nível de $\Omega$, temos $n_{e} X^{e}=0$ e $X^{e}=P_{g}^{e} X^{g}$ e portanto

$$
\begin{aligned}
\mathcal{D}_{a} \mathcal{D}_{b} X^{c}= & \mathcal{D}_{a}\left(P_{b}^{d} P_{e}^{c} \nabla_{d} X^{e}\right)=P_{a}^{f} P_{b}^{g} P_{h}^{c} \nabla_{f}\left(P_{g}^{d} P_{e}^{h} \nabla_{d} X^{e}\right) \\
= & P_{a}^{f} P_{b}^{g} P_{e}^{c} \nabla_{f}\left(\delta_{g}^{d}-n_{g} n^{d}\right) \nabla_{d} X^{e}+P_{a}^{f} P_{b}^{d} P_{h}^{c} \nabla_{f}\left(\delta_{e}^{h}-n_{e} n^{h}\right) \nabla_{d} X^{e} \\
& +P_{a}^{f} P_{b}^{d} P_{e}^{c} \nabla_{f} \nabla_{d} X^{e} \\
= & -P_{a}^{f} P_{b}^{g} P_{e}^{c}\left(\nabla_{f} n_{g}\right) n^{d} \nabla_{d} X^{e}-P_{a}^{f} P_{b}^{d} P_{h}^{c} n_{e}\left(\nabla_{f} n^{h}\right) \nabla_{d} X^{e} \\
& +P_{a}^{f} P_{b}^{d} P_{e}^{c} \nabla_{f} \nabla_{d} X^{e} \\
= & -K_{a b} P_{e}^{c} n^{d} \nabla_{d} X^{e}+K_{c}^{a} P_{b}^{d}\left(\nabla_{d} n_{e}\right) P_{g}^{e} X^{e}+P_{a}^{e} P_{b}^{f} P_{g}^{c} \nabla_{e} \nabla_{f} X^{g} \\
= & -K_{a b} P_{e}^{c} n^{d} \nabla_{d} X^{e}+K_{c}^{a} K_{b g} X^{g}+P_{a}^{e} P_{b}^{f} P_{g}^{c} \nabla_{e} \nabla_{f} X^{g} .
\end{aligned}
$$

Antisimetrizando em $a$ e $b$ e usando a fórmula (1.40), obtemos a equação (1.42). Para provar a equação (1.43), calculamos

$$
\begin{aligned}
q^{b c} \mathcal{D}_{c} K_{a b} & =q^{b c} P_{a}^{d} \nabla_{c} K_{d b}=q^{b c} P_{a}^{d} \nabla_{c}\left(P_{d}^{e} P_{b}^{f} \nabla_{e} n_{f}\right) \\
& =q^{b c} P_{a}^{d} \nabla_{c}\left(\left(\delta_{d}^{e}-n_{d} n^{e}\right)\left(\delta_{b}^{f}-n_{b} n^{f}\right) \nabla_{e} n_{f}\right) \\
& =q^{b c} P_{a}^{d} \nabla_{c} \nabla_{d} n_{b}-q^{b c} P_{a}^{d} n^{e} \nabla_{c} n_{d} \nabla_{e} n_{b} \\
& =q^{b c} P_{a}^{d} \nabla_{c} \nabla_{d} n_{b}-q^{b c} P_{a}^{d} n^{e} F^{-1 / 2} \nabla_{c} \partial_{d} \Omega \nabla_{e} n_{b}
\end{aligned}
$$

e

$$
\begin{aligned}
q^{b c} \mathcal{D}_{a} K_{c b} & =q^{b c} P_{a}^{d} \nabla_{d} K_{c b}=q^{b c} P_{a}^{d} \nabla_{d}\left(P_{c}^{e} P_{b}^{f} \nabla_{e} n_{f}\right) \\
& =q^{b c} P_{a}^{d} \nabla_{d}\left(\left(\delta_{c}^{e}-n_{c} n^{e}\right)\left(\delta_{b}^{f}-n_{b} n^{f}\right) \nabla_{e} n_{f}\right) \\
& =q^{b c} P_{a}^{d} \nabla_{d} \nabla_{c} n_{b}-q^{b c} P_{a}^{d} n^{e} \nabla_{d} n_{c} \nabla_{e} n_{b} \\
& =q^{b c} P_{a}^{d} \nabla_{d} \nabla_{c} n_{b}-q^{b c} P_{a}^{d} n^{e} F^{-1 / 2} \nabla_{d} \partial_{c} \Omega \nabla_{e} n_{b} .
\end{aligned}
$$

Logo, vale

$$
\begin{aligned}
q^{b c} \mathcal{D}_{c} K_{a b}-q^{b c} \mathcal{D}_{a} K_{c b} & =q^{b c} P_{a}^{d}\left(\nabla_{c} \nabla_{d}-\nabla_{d} \nabla_{c}\right) n_{b} \\
& =-q^{b c} P_{a}^{d} R_{b c d}^{e} n_{e} \\
& =-\left(g^{b c}-n^{b} n^{c}\right) P_{a}^{d} R_{e b c d} n^{e} \\
& =P_{a}^{d} n^{e} R_{d e}
\end{aligned}
$$

como desejado. Note ainda que contração da equação (1.42) com $P_{c}^{a}$ leva à seguinte relação envolvendo o tensor de Ricci quadridimensional e o tensor de Ricci tridimensional:

$$
\begin{aligned}
P_{a}^{c} P_{b}^{d} n_{e} n^{f} R_{c f d}^{e} & =P_{a}^{c} P_{b}^{d} \delta_{e}^{f} R_{c f d}^{e}-P_{a}^{c} P_{b}^{d} P_{e}^{f} R_{c f d}^{e} \\
& =P_{a}^{c} P_{b}^{d} R_{c d}-\mathcal{R}_{a b}+K K_{a b}-q^{c d} K_{a c} K_{b d} .
\end{aligned}
$$


Também temos, para qualquer função $F$,

$$
\begin{aligned}
\mathcal{D}_{a} \mathcal{D}_{b} F & =P_{a}^{c} P_{b}^{d} \nabla_{c}\left(P_{d}^{e} \partial_{e} F\right) \\
& =P_{a}^{c} P_{b}^{d} \nabla_{c} \partial_{d} F-P_{a}^{c} P_{b}^{d}\left(\nabla_{c} n_{d}\right) n^{e} \partial_{e} F-P_{a}^{c} P_{b}^{d} n_{d}\left(\nabla_{c} n^{e}\right) \partial_{e} F,
\end{aligned}
$$

ou seja

$$
\mathcal{D}_{a} \mathcal{D}_{b} F=P_{a}^{c} P_{b}^{d} \nabla_{c} \partial_{d} F-\left(\mathcal{L}_{n} F\right) K_{a b}
$$

de modo que, em particular,

$$
\begin{aligned}
& P_{a}^{c} P_{b}^{d}\left(\frac{3}{4} F^{-2}\left(\partial_{c} F\right)\left(\partial_{d} F\right)-\frac{1}{2} F^{-1}\left(\nabla_{c} \partial_{d} F\right)+\frac{1}{2} F^{-1}\left(\mathcal{L}_{n} F\right) K_{c d}\right) \\
& \quad=F^{1 / 2}\left(\mathcal{D}_{a} \mathcal{D}_{b} F^{-1 / 2}\right) .
\end{aligned}
$$

Agora podemos usar as equações (1.27), (1.30), (1.33), (1.35), (1.36) e (1.44) para calcular a derivada normal covariante da segunda forma fundamental como segue:

$$
\begin{aligned}
n^{c} \nabla_{c} K_{a b}= & n^{c} \nabla_{c}\left(P_{a}^{d} P_{b}^{e} \nabla_{d} n_{e}\right) \\
= & -n^{c}\left(\nabla_{c} n_{a}\right) n^{d} P_{b}^{e}\left(\nabla_{d} n_{e}\right)-n^{c} n_{a}\left(\nabla_{c} n^{d}\right) P_{b}^{e}\left(\nabla_{d} n_{e}\right) \\
& -n^{c} P_{a}^{d} n_{b}\left(\nabla_{c} n^{e}\right)\left(\nabla_{d} n_{e}\right) \\
& +P_{a}^{d} P_{b}^{e} n^{c}\left(\nabla_{d} \nabla_{c} n_{e}\right)-P_{a}^{d} P_{b}^{e} n^{c} R_{e c d}^{f} n_{f} \\
= & -\frac{1}{4} P_{a}^{c} P_{b}^{d} F^{-2}\left(\partial_{c} F\right)\left(\partial_{d} F\right) \\
& -\frac{1}{2} q^{d c} F^{-1}\left(\partial_{c} F\right) n_{a} P_{b}^{e}\left(K_{d e}+\frac{1}{2} F^{-1}\left(\partial_{f} F\right) P_{e}^{f} n_{d}\right) \\
& -\frac{1}{2} q^{e c} F^{-1}\left(\partial_{c} F\right) n_{b} P_{a}^{d}\left(K_{d e}+\frac{1}{2} F^{-1}\left(\partial_{f} F\right) P_{e}^{f} n_{d}\right) \\
& +P_{a}^{d} P_{b}^{e} \nabla_{d}\left(n^{c} \nabla_{c} n_{e}\right)-P_{a}^{d} P_{b}^{e}\left(\nabla_{d} n^{c}\right)\left(\nabla_{c} n_{e}\right) \\
& -P_{a}^{c} P_{b}^{d} n_{e} n^{f} R_{d f c}^{e} \\
= & -\frac{1}{4} P_{a}^{c} P_{b}^{d} F^{-2}\left(\partial_{c} F\right)\left(\partial_{d} F\right) \\
& -\frac{1}{2} q^{c d} F^{-1}\left(\partial_{c} F\right)\left(K_{b d} n_{a}+K_{a d} n_{b}\right) \\
& +P_{a}^{d} P_{b}^{e} \nabla_{d}\left(\frac{1}{2} F^{-1} P_{e}^{c}\left(\partial_{c} F\right)\right) \\
& -P_{a}^{d} P_{b}^{e}\left(K_{d}^{c}+\frac{1}{2} F^{-1}\left(\partial_{f} F\right) q^{f c} n_{d}\right)\left(K_{c e}+\frac{1}{2} F^{-1}\left(\partial_{f} F\right) P_{e}^{f} n_{c}\right) \\
& -P_{a}^{c} P_{b}^{d} R_{c d}+\mathcal{R}_{a b}+K K_{a b}-q^{c d} K_{a c} K_{b d}
\end{aligned}
$$




$$
\begin{aligned}
= & -\frac{1}{4} P_{a}^{c} P_{b}^{d} F^{-2}\left(\partial_{c} F\right)\left(\partial_{d} F\right) \\
& -\frac{1}{2} q^{c d} F^{-1}\left(\partial_{c} F\right)\left(K_{b d} n_{a}+K_{a d} n_{b}\right) \\
& -\frac{1}{2} P_{a}^{d} P_{b}^{c} F^{-2}\left(\partial_{c} F\right)\left(\partial_{d} F\right)-\frac{1}{2} P_{a}^{d} P_{b}^{e}\left(\nabla_{d} n_{e}\right) n^{c} F^{-1}\left(\partial_{c} F\right) \\
& +\frac{1}{2} P_{a}^{d} P_{b}^{c} F^{-1}\left(\nabla_{d} \partial_{c} F\right)-K_{b c} K_{a}^{c} \\
& -P_{a}^{c} P_{b}^{d} R_{c d}+\mathcal{R}_{a b}-K K_{a b}+q^{c d} K_{a c} K_{b d} .
\end{aligned}
$$

Assim, usando a equação (1.46), obtemos

$$
\begin{aligned}
n^{c} \nabla_{c} K_{a b}= & -\frac{1}{2} q^{c d} F^{-1}\left(\partial_{c} F\right)\left(K_{b d} n_{a}+K_{a d} n_{b}\right) \\
& -F^{1 / 2}\left(\mathcal{D}_{a} \mathcal{D}_{b} F^{-1 / 2}\right)-P_{a}^{c} P_{b}^{d} R_{c d}+\mathcal{R}_{a b}-K K_{a b},
\end{aligned}
$$

e usando a equação (1.37), concluímos que

$$
\mathcal{L}_{n} K_{a b}=-F^{1 / 2}\left(\mathcal{D}_{a} \mathcal{D}_{b} F^{-1 / 2}\right)-P_{a}^{c} P_{b}^{d} R_{c d}+\mathcal{R}_{a b}-K K_{a b}+2 q^{c d} K_{a c} K_{b d}
$$

sendo que estas fórmulas se tornarão úteis a seguir.

\subsubsection{A métrica não-física}

O passo fundamental para efetuar um reescalonamento conforme anisotrópico consiste na introdução de uma nova métrica $\hat{g}$ sobre $M$ por

$$
\hat{g}_{a b}=\Omega^{2 s} q_{a b}+\Omega^{2 t} n_{a} n_{b}, \hat{g}^{a b}=\Omega^{-2 s} q^{a b}+\Omega^{-2 t} n^{a} n^{b},
$$

onde $s$ e $t$ são parâmetros reais a serem fixados posteriormente. Assim, se definirmos

$$
\hat{n}^{a}=\hat{F}^{-1 / 2} \hat{g}^{a b} \partial_{b} \Omega
$$

$\mathrm{e}$

$$
\hat{n}_{a}=\hat{F}^{-1 / 2} \partial_{a} \Omega
$$

onde

$$
\hat{F}=\hat{g}^{a b} \partial_{a} \Omega \partial_{b} \Omega
$$

de modo que vale

$$
\hat{g}_{a b} \hat{n}^{a} \hat{n}^{b}=1=\hat{g}^{a b} \hat{n}_{a} \hat{n}_{b}
$$

e se escrevemos

$$
\hat{g}_{a b}=\hat{q}_{a b}+\hat{n}_{a} \hat{n}_{b}, \quad \hat{g}^{a b}=\hat{q}^{a b}+\hat{n}^{a} \hat{n}^{b}
$$


como antes, então temos que

$$
\begin{gathered}
\hat{q}_{a b}=\Omega^{2 s} q_{a b}, \quad \hat{q}^{a b}=\Omega^{-2 s} q^{a b}, \\
\hat{n}^{a}=\Omega^{-t} n^{a}, \quad \hat{n}_{a}=\Omega^{t} n_{a}, \\
\hat{F}=\Omega^{-2 t} F
\end{gathered}
$$

$\mathrm{e}$

$$
\hat{P}_{a}^{b}=P_{a}^{b}
$$

Neste caso, diremos que a métrica $\hat{g}$ é obtida da métrica $g$ por um reescalonamento conforme anisotrópico.

Para calcular as relações entre os símbolos de Christoffel e tensores de Riemann das duas métricas, faremos uso da fórmula geral para a diferença dos símbolos de Christoffel associados a duas métricas quaisquer $g$ e $\hat{g}$,

$$
\hat{\Gamma}_{a b}^{c}=\Gamma_{a b}^{c}+C_{a b}^{c}
$$

onde

$$
C_{a b}^{c}=\frac{1}{2} \hat{g}^{c d}\left(\nabla_{a} \hat{g}_{b d}+\nabla_{b} \hat{g}_{a d}-\nabla_{d} \hat{g}_{a b}\right)
$$

e

$$
\hat{R}_{c a b}^{d}=R_{c a b}^{d}+\nabla_{a} C_{b c}^{d}-\nabla_{b} C_{a c}^{d}+C_{a e}^{d} C_{b c}^{e}-C_{b e}^{d} C_{a c}^{e}
$$

O modo mais fácil para provar as últimas duas fórmulas baseia-se na observação de que ambos os lados são tensores e portanto é suficiente demonstrá-las, separadamente, em cada ponto da variedade e ainda apenas em algum sistema especial de coordenadas, sendo que elas se reduzem a equações bem conhecidas, tais como

$$
\hat{R}_{c a b}^{d}-R_{c a b}^{d}=\partial_{a}\left(\hat{\Gamma}_{b c}^{d}-\Gamma_{b c}^{d}\right)-\partial_{b}\left(\hat{\Gamma}_{a c}^{d}-\Gamma_{b c}^{d}\right)+\hat{\Gamma}_{a e}^{d} \hat{\Gamma}_{b c}^{e}-\hat{\Gamma}_{b e}^{d} \hat{\Gamma}_{a c}^{e}-\Gamma_{a e}^{d} \Gamma_{b c}^{e}+\Gamma_{b e}^{d} \Gamma_{a c}^{e}
$$

quando consideradas em coordenadas normais para a métrica $g$ (nas quais os símbolos de Christoffel $\Gamma_{a b}^{c}$ se anulam e as derivadas covariantes $\nabla_{a}$ coincidem com as derivadas parciais $\partial_{a}$ ). Para calcular o tensor $C$, reescrevemos a equação (1.49) na forma

$$
\hat{g}_{a b}=\Omega^{2 s} g_{a b}+\left(\Omega^{2 t}-\Omega^{2 s}\right) n_{a} n_{b}, \quad \hat{g}^{a b}=\Omega^{-2 s} g^{a b}+\left(\Omega^{-2 t}-\Omega^{-2 s}\right) n^{a} n^{b},
$$

para obter 


$$
\begin{aligned}
& 2 C_{a b}^{c}=\left(\Omega^{-2 s} g^{c d}+\left(\Omega^{-2 t}-\Omega^{-2 s}\right) n^{c} n^{d}\right) \\
& \times\left(\nabla_{a}\left(\Omega^{2 s} g_{b d}+\left(\Omega^{2 t}-\Omega^{2 s}\right) n_{b} n_{d}\right)\right. \\
& +\nabla_{b}\left(\Omega^{2 s} g_{a d}+\left(\Omega^{2 t}-\Omega^{2 s}\right) n_{a} n_{d}\right) \\
& \left.-\nabla_{d}\left(\Omega^{2 s} g_{a b}+\left(\Omega^{2 t}-\Omega^{2 s}\right) n_{a} n_{b}\right)\right) \\
& =\left(\Omega^{-2 s} q^{c d}+\Omega^{-2 t} n^{c} n^{d}\right) \\
& \times\left(2 s \Omega^{2 s-1} F^{1 / 2} n_{a} g_{b d}+\left(2 t \Omega^{2 t-1}-2 s \Omega^{2 s-1}\right) F^{1 / 2} n_{a} n_{b} n_{d}\right. \\
& +\left(\Omega^{2 t}-\Omega^{2 s}\right)\left(\left(\nabla_{a} n_{b}\right) n_{d}+n_{b}\left(\nabla_{a} n_{d}\right)\right) \\
& +2 s \Omega^{2 s-1} F^{1 / 2} n_{b} g_{a d}+\left(2 t \Omega^{2 t-1}-2 s \Omega^{2 s-1}\right) F^{1 / 2} n_{a} n_{b} n_{d} \\
& +\left(\Omega^{2 t}-\Omega^{2 s}\right)\left(\left(\nabla_{b} n_{a}\right) n_{d}+n_{a}\left(\nabla_{b} n_{d}\right)\right) \\
& -2 s \Omega^{2 s-1} F^{1 / 2} n_{d} g_{a b}-\left(2 t \Omega^{2 t-1}-2 s \Omega^{2 s-1}\right) F^{1 / 2} n_{a} n_{b} n_{d} \\
& \left.-\left(\Omega^{2 t}-\Omega^{2 s}\right)\left(\left(\nabla_{d} n_{a}\right) n_{b}+n_{a}\left(\nabla_{d} n_{b}\right)\right)\right) \\
& =2 s \Omega^{-1} F^{1 / 2} P_{b}^{c} n_{a}+2 s \Omega^{2 s-2 t-1} F^{1 / 2} n_{a} n_{b} n^{c} \\
& +\left(2 t-2 s \Omega^{2 s-2 t}\right) \Omega^{-1} F^{1 / 2} n_{a} n_{b} n^{c} \\
& -\left(1-\Omega^{2 t-2 s}\right) q^{c d} n_{b}\left(\nabla_{a} n_{d}\right)+\left(1-\Omega^{2 s-2 t}\right)\left(\nabla_{a} n_{b}\right) n^{c} \\
& +2 s \Omega^{-1} F^{1 / 2} P_{a}^{c} n_{b}+2 s \Omega^{2 s-2 t-1} F^{1 / 2} n_{a} n_{b} n^{c} \\
& +\left(2 t-2 s \Omega^{2 s-2 t}\right) \Omega^{-1} F^{1 / 2} n_{a} n_{b} n^{c} \\
& -\left(1-\Omega^{2 t-2 s}\right) q^{c d} n_{a}\left(\nabla_{b} n_{d}\right)+\left(1-\Omega^{2 s-2 t}\right)\left(\nabla_{b} n_{a}\right) n^{c} \\
& -2 s \Omega^{2 s-2 t-1} F^{1 / 2} g_{a b} n^{c}-\left(2 t-2 s \Omega^{2 s-2 t}\right) \Omega^{-1} F^{1 / 2} n_{a} n_{b} n^{c} \\
& +\left(1-\Omega^{2 t-2 s}\right) q^{c d}\left(\left(\nabla_{d} n_{a}\right) n_{b}+n_{a}\left(\nabla_{d} n_{b}\right)\right) \\
& -\left(1-\Omega^{2 s-2 t}\right)\left(n^{d}\left(\nabla_{d} n_{a}\right) n_{b} n^{c}+n_{a} n^{d}\left(\nabla_{d} n_{b}\right) n^{c}\right) \\
& =2 s \Omega^{-1} F^{1 / 2}\left(P_{a}^{c} n_{b}+P_{b}^{c} n_{a}\right)+2 t \Omega^{-1} F^{1 / 2} n_{a} n_{b} n^{c}-2 s \Omega^{2 s-2 t-1} F^{1 / 2} q_{a b} n^{c} \\
& \left.+\left(1-\Omega^{2 t-2 s}\right) q^{c d}\left(\left(\nabla_{d} n_{a}-\nabla_{a} n_{d}\right) n_{b}+n_{a}\left(\nabla_{d} n_{b}-\nabla_{b} n_{d}\right)\right)\right) \\
& +\left(1-\Omega^{2 s-2 t}\right)\left(\nabla_{a} n_{b}+\nabla_{b} n_{a}-n^{d}\left(\nabla_{d} n_{a}\right) n_{b}-n_{a} n^{d}\left(\nabla_{d} n_{b}\right)\right) n^{c}
\end{aligned}
$$




$$
\begin{aligned}
&= 2 s \Omega^{-1} F^{1 / 2}\left(P_{a}^{c} n_{b}+P_{b}^{c} n_{a}\right)+2 t \Omega^{-1} F^{1 / 2} n_{a} n_{b} n^{c} \\
&-2 s \Omega^{2 s-2 t-1} F^{1 / 2} q_{a b} n^{c}+2\left(1-\Omega^{2 s-2 t}\right) K_{a b} n^{c} \\
&-\left(1-\Omega^{2 t-2 s}\right) q^{c d}\left(\left(n_{d} n^{e}\left(\nabla_{e} n_{a}\right)-n_{a} n^{e}\left(\nabla_{e} n_{d}\right)\right) n_{b}\right. \\
&\left.+n_{a}\left(n_{d} n^{e}\left(\nabla_{e} n_{b}\right)-n_{b} n^{e}\left(\nabla_{e} n_{d}\right)\right)\right)
\end{aligned}
$$

ou seja,

$$
\begin{aligned}
C_{a b}^{c}= & s \Omega^{-1} F^{1 / 2}\left(P_{a}^{c} n_{b}+P_{b}^{c} n_{a}\right)+t \Omega^{-1} F^{1 / 2} n_{a} n_{b} n^{c} \\
& -s \Omega^{2 s-2 t-1} F^{1 / 2} q_{a b} n^{c}+\left(1-\Omega^{2 s-2 t}\right) K_{a b} n^{c} \\
& -\frac{1}{2}\left(1-\Omega^{2 t-2 s}\right) F^{-1}\left(\partial_{d} F\right) q^{c d} n_{a} n_{b} .
\end{aligned}
$$

Como corolário, notamos que

$$
\begin{aligned}
\hat{K}_{a b} & =\hat{P}_{a}^{c} \hat{P}_{b}^{d} \hat{\nabla}_{c} \hat{n}_{d}=\hat{P}_{a}^{c} \hat{P}_{b}^{d}\left(\nabla_{c}\left(\Omega^{t} n_{d}\right)-C_{c d}^{e}\left(\Omega^{t} n_{e}\right)\right) \\
& =\Omega^{t} P_{a}^{c} P_{b}^{d} \nabla_{c} n_{d}+s \Omega^{2 s-t-1} F^{1 / 2} q_{a b}-\Omega^{t}\left(1-\Omega^{2 s-2 t}\right) K_{a b},
\end{aligned}
$$

o que leva à seguinte fórmula para a relação entre as segundas formas fundamentais das duas métricas:

$$
\hat{K}_{a b}=\Omega^{2 s-t} K_{a b}+s \Omega^{2 s-t-1} F^{1 / 2} q_{a b} .
$$

Para calcular a relação entre os tensores de Ricci e, posteriormente, as curvaturas escalares e os tensores de Einstein das duas métricas, começamos por contrair os índices $a$ e $d$ na equação (1.61) para obter

$$
\hat{R}_{a b}=R_{a b}+\nabla_{c} C_{b a}^{c}-\nabla_{b} C_{c a}^{c}+C_{c d}^{c} C_{b a}^{d}-C_{b d}^{c} C_{c a}^{d} .
$$

Inserindo a equação (1.63) e usando as equações (1.31)-(1.33), vem

$$
\begin{aligned}
\nabla_{c} C_{b a}^{c}= & \nabla_{c}\left(s \Omega^{-1} F^{1 / 2}\left(P_{b}^{c} n_{a}+P_{a}^{c} n_{b}\right)+t \Omega^{-1} F^{1 / 2} n_{a} n_{b} n^{c}-s \Omega^{2 s-2 t-1} F^{1 / 2} q_{a b} n^{c}\right. \\
& \left.\quad+\left(1-\Omega^{2 s-2 t}\right) K_{a b} n^{c}-\frac{1}{2}\left(1-\Omega^{2 t-2 s}\right) F^{-1}\left(\partial_{d} F\right) q^{c d} n_{a} n_{b}\right) \\
= & -s \Omega^{-2}\left(\partial_{c} \Omega\right) F^{1 / 2} P_{b}^{c} n_{a}-s \Omega^{-2}\left(\partial_{c} \Omega\right) F^{1 / 2} P_{a}^{c} n_{b} \\
& +\frac{1}{2} s \Omega^{-1} F^{-1 / 2}\left(\partial_{c} F\right) P_{b}^{c} n_{a}+\frac{1}{2} s \Omega^{-1} F^{-1 / 2}\left(\partial_{c} F\right) P_{a}^{c} n_{b} \\
& -s \Omega^{-1} F^{1 / 2}\left(\nabla_{c} n_{b}\right) n^{c} n_{a}-s \Omega^{-1} F^{1 / 2} K n_{a} n_{b}+s \Omega^{-1} F^{1 / 2} P_{b}^{c}\left(\nabla_{c} n_{a}\right) \\
& -s \Omega^{-1} F^{1 / 2}\left(\nabla_{c} n_{a}\right) n^{c} n_{b}-s \Omega^{-1} F^{1 / 2} K n_{a} n_{b}+s \Omega^{-1} F^{1 / 2} P_{a}^{c}\left(\nabla_{c} n_{d}\right) \\
& -t \Omega^{-2} F n_{a} n_{b}+\frac{1}{2} t \Omega^{-1} F^{-1 / 2}\left(\partial_{c} F\right) n_{a} n_{b} n^{c} \\
& +t \Omega^{-1} F^{1 / 2}\left(\nabla_{c} n_{a}\right) n_{b} n^{c}+t \Omega^{-1} F^{1 / 2}\left(\nabla_{c} n_{b}\right) n_{a} n^{c}+t \Omega^{-1} F^{1 / 2} K n_{a} n_{b}
\end{aligned}
$$




$$
\begin{aligned}
& -s(2 s-2 t-1) \Omega^{2 s-2 t-2} F q_{a b}-\frac{1}{2} s \Omega^{2 s-2 t-1} F^{-1 / 2}\left(\partial_{c} F\right) q_{a b} n^{c} \\
& +s \Omega^{2 s-2 t-1} F^{1 / 2}\left(\nabla_{c} n_{a}\right) n_{b} n^{c}+s \Omega^{2 s-2 t-1} F^{1 / 2}\left(\nabla_{c} n_{b}\right) n_{a} n^{c} \\
& -s \Omega^{2 s-2 t-1} F^{1 / 2} K q_{a b}-(2 s-2 t) \Omega^{2 s-2 t-1} F^{1 / 2} K_{a b} \\
& +\left(1-\Omega^{2 s-2 t}\right)\left(\nabla_{c} K_{a b}\right) n^{c}+\left(1-\Omega^{2 s-2 t}\right) K K_{a b} \\
& +(t-s) \Omega^{2 t-2 s-1}\left(\partial_{c} \Omega\right) F^{-1}\left(\partial_{d} F\right) q^{c d} n_{a} n_{b} \\
& +\frac{1}{2}\left(1-\Omega^{2 t-2 s}\right) F^{-2}\left(\partial_{c} F\right)\left(\partial_{d} F\right) q^{c d} n_{a} n_{b} \\
& -\frac{1}{2}\left(1-\Omega^{2 t-2 s}\right) F^{-1}\left(\nabla_{c} \partial_{d} F\right) q^{c d} n_{a} n_{b} \\
& +\frac{1}{2}\left(1-\Omega^{2 t-2 s}\right) F^{-1}\left(\partial_{d} F\right) K n_{a} n_{b} n^{d} \\
& +\frac{1}{2}\left(1-\Omega^{2 t-2 s}\right) F^{-1}\left(\partial_{d} F\right) g^{d e}\left(\nabla_{c} n_{e}\right) n^{c} n_{a} n_{b} \\
& -\frac{1}{2}\left(1-\Omega^{2 t-2 s}\right) F^{-1}\left(\partial_{d} F\right) q^{c d}\left(\nabla_{c} n_{a}\right) n_{b} \\
& -\frac{1}{2}\left(1-\Omega^{2 t-2 s}\right) F^{-1}\left(\partial_{d} F\right) q^{c d}\left(\nabla_{c} n_{b}\right) n_{a} .
\end{aligned}
$$

Observamos que os termos na primeira e na décima primeira linha se anulam e que devido à equação (1.35), os termos na segunda linha cancelam os primeiros termos na terceira e na quarta linha. Usando as equações (1.35) e (1.36) para reescrever os termos contendo derivadas covariantes de $n$, obtemos

$$
\begin{aligned}
\nabla_{c} C_{b a}^{c}= & -(2 s-t) \Omega^{-1} F^{1 / 2} K n_{a} n_{b}+2 s \Omega^{-1} F^{1 / 2} K_{a b} \\
& -t \Omega^{-2} F n_{a} n_{b}+\frac{1}{2} t \Omega^{-1} F^{-1 / 2}\left(\mathcal{L}_{n} F\right) n_{a} n_{b} \\
& +\frac{1}{2} t \Omega^{-1} F^{-1 / 2}\left(\partial_{c} F\right)\left(P_{a}^{c} n_{b}+P_{b}^{c} n_{a}\right) \\
& -s(2 s-2 t-1) \Omega^{2 s-2 t-2} F q_{a b}-\frac{1}{2} s \Omega^{2 s-2 t-1} F^{-1 / 2}\left(\mathcal{L}_{n} F\right) q_{a b} \\
& +\frac{1}{2} s \Omega^{2 s-2 t-1} F^{-1 / 2}\left(\partial_{c} F\right)\left(P_{a}^{c} n_{b}+P_{b}^{c} n_{a}\right) \\
& -s \Omega^{2 s-2 t-1} F^{1 / 2} K q_{a b}-(2 s-2 t) \Omega^{2 s-2 t-1} F^{1 / 2} K_{a b} \\
& +\left(1-\Omega^{2 s-2 t}\right) K K_{a b}+\left(1-\Omega^{2 s-2 t}\right) n^{c}\left(\nabla_{c} K_{a b}\right) \\
& +\frac{3}{4}\left(1-\Omega^{2 t-2 s}\right) F^{-2}\left(\partial_{c} F\right)\left(\partial_{d} F\right) q^{c d} n_{a} n_{b} \\
& -\frac{1}{2}\left(1-\Omega^{2 t-2 s}\right) F^{-1}\left(\nabla_{c} \partial_{d} F\right) q^{c d} n_{a} n_{b} \\
& +\frac{1}{2}\left(1-\Omega^{2 t-2 s}\right) F^{-1}\left(\mathcal{L}_{n} F\right) K n_{a} n_{b} \\
& -\frac{1}{2}\left(1-\Omega^{2 t-2 s}\right) F^{-1}\left(\partial_{d} F\right) q^{c d}\left(K_{a c} n_{b}+K_{b c} n_{a}\right) .
\end{aligned}
$$

De maneira análoga,

$$
\begin{gathered}
\nabla_{b} C_{c a}^{c}=\nabla_{b}\left(s \Omega^{-1} F^{1 / 2}\left(P_{c}^{c} n_{a}+P_{a}^{c} n_{c}\right)+t \Omega^{-1} F^{1 / 2} n_{a} n_{c} n^{c}-s \Omega^{2 s-2 t-1} F^{1 / 2} q_{a c} n^{c}\right. \\
\left.+\left(1-\Omega^{2 s-2 t}\right) K_{a c} n^{c}-\frac{1}{2}\left(1-\Omega^{2 t-2 s}\right) F^{-1}\left(\partial_{d} F\right) q^{c d} n_{a} n_{c}\right)
\end{gathered}
$$




$$
\begin{aligned}
= & (3 s+t) \nabla_{b}\left(\Omega^{-1} F^{1 / 2} n_{a}\right) \\
= & -(3 s+t) \Omega^{-2} F n_{a} n_{b}+\frac{1}{2}(3 s+t) \Omega^{-1} F^{-1 / 2}\left(\partial_{c} F\right)\left(P_{b}^{c}+n_{b} n^{c}\right) n_{a} \\
& +(3 s+t) \Omega^{-1} F^{1 / 2}\left(\nabla_{b} n_{a}\right) \\
= & -(3 s+t) \Omega^{-2} F n_{a} n_{b}+\frac{1}{2}(3 s+t) \Omega^{-1} F^{-1 / 2}\left(\partial_{c} F\right)\left(P_{a}^{c} n_{b}+P_{b}^{c} n_{a}\right) \\
& +\frac{1}{2}(3 s+t) \Omega^{-1} F^{-1 / 2}\left(\mathcal{L}_{n} F\right) n_{a} n_{b}+(3 s+t) \Omega^{-1} F^{1 / 2} K_{a b} .
\end{aligned}
$$

Finalmente, temos

$$
\begin{aligned}
& C_{c d}^{c} C_{b a}^{d}=\left(s \Omega^{-1} F^{1 / 2}\left(P_{c}^{c} n_{d}+P_{d}^{c} n_{c}\right)+t \Omega^{-1} F^{1 / 2} n_{c} n_{d} n^{c}-s \Omega^{2 s-2 t-1} F^{1 / 2} q_{c d} n^{c}\right. \\
& \left.+\left(1-\Omega^{2 s-2 t}\right) K_{c d} n^{c}-\frac{1}{2}\left(1-\Omega^{2 t-2 s}\right) F^{-1}\left(\partial_{e} F\right) q^{e c} n_{c} n_{d}\right) \\
& \times\left(s \Omega^{-1} F^{1 / 2}\left(P_{b}^{d} n_{a}+P_{a}^{d} n_{b}\right)+t \Omega^{-1} F^{1 / 2} n_{a} n_{b} n^{d}-s \Omega^{2 s-2 t-1} F^{1 / 2} q_{a b} n^{d}\right. \\
& \left.+\left(1-\Omega^{2 s-2 t}\right) K_{a b} n^{d}-\frac{1}{2}\left(1-\Omega^{2 t-2 s}\right) F^{-1}\left(\partial_{f} F\right) q^{f d} n_{a} n_{b}\right) \\
& =(3 s+t) \Omega^{-1} F^{1 / 2} n_{d} \\
& \times\left(s \Omega^{-1} F^{1 / 2}\left(P_{b}^{d} n_{a}+P_{a}^{d} n_{b}\right)+t \Omega^{-1} F^{1 / 2} n_{a} n_{b} n^{d}-s \Omega^{2 s-2 t-1} F^{1 / 2} q_{a b} n^{d}\right. \\
& \left.+\left(1-\Omega^{2 s-2 t}\right) K_{a b} n^{d}-\frac{1}{2}\left(1-\Omega^{2 t-2 s}\right) F^{-1}\left(\partial_{f} F\right) q^{f d} n_{a} n_{b}\right) \\
& =t(3 s+t) \Omega^{-2} F n_{a} n_{b}-s(3 s+t) \Omega^{2 s-2 t-2} F q_{a b} \\
& +(3 s+t) \Omega^{-1}\left(1-\Omega^{2 s-2 t}\right) F^{1 / 2} K_{a b},
\end{aligned}
$$

e

$$
\begin{aligned}
C_{b d}^{c} C_{c a}^{d}= & \left(s \Omega^{-1} F^{1 / 2}\left(P_{b}^{c} n_{d}+P_{d}^{c} n_{b}\right)+t \Omega^{-1} F^{1 / 2} n_{b} n_{d} n^{c}-s \Omega^{2 s-2 t-1} F^{1 / 2} q_{b d} n^{c}\right. \\
& \left.+\left(1-\Omega^{2 s-2 t}\right) K_{b d} n^{c}-\frac{1}{2}\left(1-\Omega^{2 t-2 s}\right) F^{-1}\left(\partial_{e} F\right) q^{e c} n_{b} n_{d}\right) \\
& \times\left(s \Omega^{-1} F^{1 / 2}\left(P_{c}^{d} n_{a}+P_{a}^{d} n_{c}\right)+t \Omega^{-1} F^{1 / 2} n_{a} n_{c} n^{d}-s \Omega^{2 s-2 t-1} F^{1 / 2} q_{a c} n^{d}\right. \\
& \left.\quad+\left(1-\Omega^{2 s-2 t}\right) K_{a c} n^{d}-\frac{1}{2}\left(1-\Omega^{2 t-2 s}\right) F^{-1}\left(\partial_{f} F\right) q^{f d} n_{a} n_{c}\right) \\
= & \left(3 s^{2}+t^{2}\right) \Omega^{-2} F n_{a} n_{b}-2 s^{2} \Omega^{2 s-2 t-2} F q_{a b}+2 s \Omega^{-1}\left(1-\Omega^{2 s-2 t}\right) F^{1 / 2} K_{a b} \\
& -\frac{1}{2} s \Omega^{-1}\left(1-\Omega^{2 s-2 t}\right) F^{-1 / 2}\left(\partial_{c} F\right)\left(P_{a}^{c} n_{b}+P_{b}^{c} n_{a}\right) \\
& -\frac{1}{2}\left(1-\Omega^{2 s-2 t}\right)\left(1-\Omega^{2 t-2 s}\right) F^{-1}\left(\partial_{c} F\right) q^{c d}\left(K_{a d} n_{b}+K_{b d} n_{a}\right) .
\end{aligned}
$$


Combinando estes resultados, obtemos

$$
\begin{aligned}
\hat{R}_{a b}= & R_{a b}-(2 s-t) \Omega^{-1} F^{1 / 2} K n_{a} n_{b}-3 s(s-t-1) \Omega^{-2} F n_{a} n_{b} \\
& -\frac{3}{2} s \Omega^{-1} F^{-1 / 2}\left(\mathcal{L}_{n} F\right) n_{a} n_{b} \\
& +\frac{3}{4}\left(1-\Omega^{2 t-2 s}\right) F^{-2}\left(\partial_{c} F\right)\left(\partial_{d} F\right) q^{c d} n_{a} n_{b} \\
& -\frac{1}{2}\left(1-\Omega^{2 t-2 s}\right) F^{-1}\left(\nabla_{c} \partial_{d} F\right) q^{c d} n_{a} n_{b} \\
& +\frac{1}{2}\left(1-\Omega^{2 t-2 s}\right) F^{-1}\left(\mathcal{L}_{n} F\right) K n_{a} n_{b} \\
& +\frac{1}{2}\left(1-\Omega^{2 s-2 t}\right) F^{-1}\left(\partial_{c} F\right) q^{c d}\left(K_{a d} n_{b}+K_{b d} n_{a}\right) \\
& -s \Omega^{-1} F^{-1 / 2}\left(\partial_{c} F\right)\left(P_{a}^{c} n_{b}+P_{b}^{c} n_{a}\right) \\
& -s(3 s-t-1) \Omega^{2 s-2 t-2} F q_{a b}-\frac{1}{2} s \Omega^{2 s-2 t-1} F^{-1 / 2}\left(\mathcal{L}_{n} F\right) q_{a b} \\
& -s \Omega^{2 s-2 t-1} F^{1 / 2} K q_{a b}-(3 s-t) \Omega^{2 s-2 t-1} F^{1 / 2} K_{a b} \\
& +\left(1-\Omega^{2 s-2 t}\right) K K_{a b}+\left(1-\Omega^{2 s-2 t}\right) n^{c}\left(\nabla_{c} K_{a b}\right) .
\end{aligned}
$$

Inserindo as equações (1.46) e (1.47) e separando as componentes puramente normais, mistas e puramente tangenciais, concluímos que

$$
\begin{aligned}
\hat{R}_{a b}= & \left(n^{c} n^{d} R_{c d}-(2 s-t) \Omega^{-1} F^{1 / 2} K-3 s(s-t-1) \Omega^{-2} F\right. \\
& \left.-\frac{3}{2} s \Omega^{-1} F^{-1 / 2}\left(\mathcal{L}_{n} F\right)+\left(1-\Omega^{2 t-2 s}\right) q^{c d} F^{1 / 2}\left(\mathcal{D}_{c} \mathcal{D}_{d} F^{-1 / 2}\right)\right) n_{a} n_{b} \\
+ & \left(R_{c d}-s \Omega^{-1} F^{-1 / 2}\left(\left(\partial_{c} F\right) n_{d}+\left(\partial_{d} F\right) n_{c}\right)\right)\left(P_{a}^{c} n_{b} n^{d}+P_{b}^{d} n_{a} n^{c}\right) \\
+ & \left(\Omega^{2 s-2 t} R_{c d}+\left(1-\Omega^{2 s-2 t}\right) \mathcal{R}_{c d}-s(3 s-t-1) \Omega^{2 s-2 t-2} F q_{c d}\right. \\
& \quad-\frac{1}{2} s \Omega^{2 s-2 t-1} F^{-1 / 2}\left(\mathcal{L}_{n} F\right) q_{c d}-s \Omega^{2 s-2 t-1} F^{1 / 2} K q_{c d} \\
& \left.\quad(3 s-t) \Omega^{2 s-2 t-1} F^{1 / 2} K_{c d}-\left(1-\Omega^{2 s-2 t}\right) F^{1 / 2}\left(\mathcal{D}_{c} \mathcal{D}_{d} F^{-1 / 2}\right)\right) P_{a}^{c} P_{b}^{d} .
\end{aligned}
$$

Agora é fácil deduzir a relação análoga entre os correspondentes tensores de Einstein $\hat{G}_{a b}$ e $G_{a b}$, usando que

$$
\begin{aligned}
\hat{n}^{a} \hat{n}^{b} \hat{G}_{a b} & =\hat{n}^{a} \hat{n}^{b}\left(\hat{R}_{a b}-\frac{1}{2} \hat{R} \hat{g}_{a b}\right)=\hat{n}^{a} \hat{n}^{b} \hat{R}_{a b}-\frac{1}{2} \hat{R}=\hat{n}^{a} \hat{n}^{b} \hat{R}_{a b}-\frac{1}{2} \hat{g}^{a b} \hat{R}_{a b} \\
& =\hat{n}^{a} \hat{n}^{b} \hat{R}_{a b}-\frac{1}{2}\left(\hat{n}^{a} \hat{n}^{b}+\hat{q}^{a b}\right) \hat{R}_{a b}=\frac{1}{2}\left(\hat{n}^{a} \hat{n}^{b} \hat{R}_{a b}-\hat{q}^{a b} \hat{R}_{a b}\right) \\
\hat{P}_{a}^{c} \hat{P}_{b}^{d} \hat{G}_{c d} & =\hat{P}_{a}^{c} \hat{P}_{b}^{d}\left(\hat{R}_{c d}-\frac{1}{2} \hat{R} \hat{g}_{c d}\right)=\hat{P}_{a}^{c} \hat{P}_{b}^{d} \hat{R}_{c d}-\frac{1}{2} \hat{R} \hat{q}_{a b}=\hat{P}_{a}^{c} \hat{P}_{b}^{d} \hat{R}_{c d}-\frac{1}{2} \hat{g}^{c d} \hat{R}_{c d} \hat{q}_{a b} \\
& =\hat{P}_{a}^{c} \hat{P}_{b}^{d} \hat{R}_{c d}-\frac{1}{2}\left(\hat{n}^{c} \hat{n}^{d} \hat{R}_{c d}+\hat{q}^{c d} \hat{R}_{c d}\right) \hat{q}_{a b} .
\end{aligned}
$$


Assim,

$$
\begin{aligned}
\hat{n}^{a} \hat{n}^{b} \hat{G}_{a b}=\frac{1}{2}( & \Omega^{-2 t} n^{a} n^{b} R_{a b}-(2 s-t) \Omega^{-2 t-1} F^{1 / 2} K-3 s(s-t-1) \Omega^{-2 t-2} F \\
& -\frac{3}{2} s \Omega^{-2 t-1} F^{-1 / 2}\left(\mathcal{L}_{n} F\right)+\left(\Omega^{-2 t}-\Omega^{-2 s}\right) F^{1 / 2} q^{a b}\left(\mathcal{D}_{a} \mathcal{D}_{b} F^{-1 / 2}\right) \\
& -\Omega^{-2 t} q^{a b} R_{a b}-\left(\Omega^{-2 s}-\Omega^{-2 t}\right) \mathcal{R}+3 s(3 s-t-1) \Omega^{-2 t-2} F \\
& +\frac{3}{2} s \Omega^{-2 t-1} F^{-1 / 2}\left(\mathcal{L}_{n} F\right)+(6 s-t) \Omega^{-2 t-1} F^{1 / 2} K \\
& \left.+\left(\Omega^{-2 s}-\Omega^{-2 t}\right) q^{a b} F^{1 / 2}\left(\mathcal{D}_{a} \mathcal{D}_{b} F^{-1 / 2}\right)\right)
\end{aligned}
$$

e analogamente

$$
\begin{aligned}
\hat{P}_{a}^{c} \hat{P}_{b}^{d} \hat{G}_{c d}= & \Omega^{2 s-2 t} P_{a}^{c} P_{b}^{d} R_{c d}+\left(1-\Omega^{2 s-2 t}\right) \mathcal{R}_{a b}-s(3 s-t-1) \Omega^{2 s-2 t-2} F q_{a b} \\
& -\frac{1}{2} s \Omega^{2 s-2 t-1} F^{-1 / 2}\left(\mathcal{L}_{n} F\right) q_{a b}-s \Omega^{2 s-2 t-1} F^{1 / 2} K q_{a b} \\
& -(3 s-t) \Omega^{2 s-2 t-1} F^{1 / 2} K_{a b}-\left(1-\Omega^{2 s-2 t}\right) F^{1 / 2}\left(\mathcal{D}_{a} \mathcal{D}_{b} F^{-1 / 2}\right) \\
& -\frac{1}{2} \Omega^{2 s}\left(\Omega^{-2 t} n^{c} n^{d} R_{c d}-(2 s-t) \Omega^{-2 t-1} F^{1 / 2} K-3 s(s-t-1) \Omega^{-2 t-2} F\right. \\
& -\frac{3}{2} s \Omega^{-2 t-1} F^{-1 / 2}\left(\mathcal{L}_{n} F\right)+\left(\Omega^{-2 t}-\Omega^{-2 s}\right) F^{1 / 2} q^{c d}\left(\mathcal{D}_{c} \mathcal{D}_{d} F^{-1 / 2}\right) \\
& +\Omega^{-2 t} q^{c d} R_{c d}+\left(\Omega^{-2 s}-\Omega^{-2 t}\right) \mathcal{R}-3 s(3 s-t-1) \Omega^{-2 t-2} F \\
& -\frac{3}{2} s \Omega^{-2 t-1} F^{-1 / 2}\left(\mathcal{L}_{n} F\right)-(6 s-t) \Omega^{-2 t-1} F^{1 / 2} K \\
& \left.-\left(\Omega^{-2 s}-\Omega^{-2 t}\right) q^{c d} F^{1 / 2}\left(\mathcal{D}_{c} \mathcal{D}_{d} F^{-1 / 2}\right)\right) q_{a b} .
\end{aligned}
$$

O resultado é

$$
\begin{aligned}
\hat{n}^{a} \hat{n}^{b} \hat{G}_{a b}= & \Omega^{-2 t} n^{a} n^{b} G_{a b}-\frac{1}{2}\left(\Omega^{-2 s}-\Omega^{-2 t}\right) \mathcal{R} \\
& +2 s \Omega^{-2 t-1} F^{1 / 2} K+3 s^{2} \Omega^{-2 t-2} F
\end{aligned}
$$

e

$$
\begin{aligned}
\hat{P}_{a}^{c} \hat{P}_{b}^{d} \hat{G}_{c d}= & \Omega^{2 s-2 t} P_{a}^{c} P_{b}^{d} G_{c d}+\left(1-\Omega^{2 s-2 t}\right)\left(\mathcal{R}_{a b}-\frac{1}{2} \mathcal{R} q_{a b}\right) \\
& -(3 s-t) \Omega^{2 s-2 t-1} F^{1 / 2}\left(K_{a b}-K q_{a b}\right) \\
& +s(3 s-2 t-2) \Omega^{2 s-2 t-2} F q_{a b}+s \Omega^{2 s-2 t-1} F^{-1 / 2}\left(\mathcal{L}_{n} F\right) q_{a b} \\
& -\left(1-\Omega^{2 s-2 t}\right)\left(F^{1 / 2}\left(\mathcal{D}_{a} \mathcal{D}_{b} F^{-1 / 2}\right)-q^{c d} F^{1 / 2}\left(\mathcal{D}_{c} \mathcal{D}_{d} F^{-1 / 2}\right) q_{a b}\right)
\end{aligned}
$$


Ainda podemos reescrever as relações deduzidas acima exclusivamente em termos de quantidades que permanecem regulares no limite $\Omega \rightarrow 0$. Para tanto, usamos que os argumentos utilizados e os cálculos efetuados até agora são simétricos sob a troca $g \leftrightarrow \hat{g}$ das duas métricas e de todas as quantidades derivadas, acompanhada por uma mudança de sinal dos parâmetros $s$ e $t$. Assim, temos como recíproca da equação (1.66),

$$
\begin{aligned}
R_{a b}=( & \hat{n}^{c} \hat{n}^{d} \hat{R}_{c d}+(2 s-t) \Omega^{-1} \hat{F}^{1 / 2} \hat{K}-3 s(s-t+1) \Omega^{-2} \hat{F} \\
& \left.+\frac{3}{2} s \Omega^{-1} \hat{F}^{-1 / 2}\left(\mathcal{L}_{\hat{n}} \hat{F}\right)+\left(1-\Omega^{2 s-2 t}\right) \hat{q}^{c d} \hat{F}^{1 / 2}\left(\hat{\mathcal{D}}_{c} \hat{\mathcal{D}}_{d} \hat{F}^{-1 / 2}\right)\right) \hat{n}_{a} \hat{n}_{b} \\
+ & \left(\hat{R}_{c d}+s \Omega^{-1} \hat{F}^{-1 / 2}\left(\left(\partial_{c} \hat{F}\right) \hat{n}_{d}+\left(\partial_{d} \hat{F}\right) \hat{n}_{c}\right)\right)\left(\hat{P}_{a}^{c} \hat{n}_{b} \hat{n}^{d}+\hat{P}_{b}^{d} \hat{n}_{a} \hat{n}^{c}\right) \\
+ & \left(\Omega^{2 t-2 s} \hat{R}_{c d}+\left(1-\Omega^{2 t-2 s}\right) \hat{\mathcal{R}}_{c d}-s(3 s-t+1) \Omega^{2 t-2 s-2} \hat{F} \hat{q}_{c d}\right. \\
& \quad+\frac{1}{2} s \Omega^{2 t-2 s-1} \hat{F}^{-1 / 2}\left(\mathcal{L}_{\hat{n}} \hat{F}\right) \hat{q}_{c d}+s \Omega^{2 t-2 s-1} \hat{F}^{1 / 2} \hat{K} \hat{q}_{c d} \\
& \left.+(3 s-t) \Omega^{2 t-2 s-1} \hat{F}^{1 / 2} \hat{K}_{c d}-\left(1-\Omega^{2 t-2 s}\right) \hat{F}^{1 / 2}\left(\hat{\mathcal{D}}_{c} \hat{\mathcal{D}}_{d} \hat{F}^{-1 / 2}\right)\right) \hat{P}_{a}^{c} \hat{P}_{b}^{d} .
\end{aligned}
$$

De maneira semelhante, temos como recíproca da equação (1.67)

$$
\begin{aligned}
n^{a} n^{b} G_{a b}= & \Omega^{2 t} \hat{n}^{a} \hat{n}^{b} \hat{G}_{a b}-\frac{1}{2}\left(\Omega^{2 s}-\Omega^{2 t}\right) \hat{\mathcal{R}} \\
& -2 s \Omega^{2 t-1} \hat{F}^{1 / 2} \hat{K}+3 s^{2} \Omega^{2 t-2} \hat{F}
\end{aligned}
$$

e como recíproca da equação (1.68)

$$
\begin{aligned}
P_{a}^{c} P_{b}^{d} G_{c d}= & \Omega^{2 t-2 s} \hat{P}_{a}^{c} \hat{P}_{b}^{d} \hat{G}_{c d}+\left(1-\Omega^{2 t-2 s}\right)\left(\hat{\mathcal{R}}_{a b}-\frac{1}{2} \hat{\mathcal{R}} \hat{q}_{a b}\right) \\
& +(3 s-t) \Omega^{2 t-2 s-1} \hat{F}^{1 / 2}\left(\hat{K}_{a b}-\hat{K} \hat{q}_{a b}\right) \\
& +s(3 s-2 t+2) \Omega^{2 t-2 s-2} \hat{F} \hat{q}_{a b}-s \Omega^{2 t-2 s-1} \hat{F}^{-1 / 2}\left(\mathcal{L}_{\hat{n}} \hat{F}\right) \hat{q}_{a b} \\
& -\left(1-\Omega^{2 t-2 s}\right)\left(\hat{F}^{1 / 2}\left(\hat{\mathcal{D}}_{a} \hat{\mathcal{D}}_{b} \hat{F}^{-1 / 2}\right)-\hat{q}^{c d} \hat{F}^{1 / 2}\left(\hat{\mathcal{D}}_{c} \hat{\mathcal{D}}_{d} \hat{F}^{-1 / 2}\right) \hat{q}_{a b}\right)
\end{aligned}
$$

\subsection{Espaços-tempos assintoticamente planos}

Com os resultados dos cálculos da seção anterior à nossa disposição, estamos prontos para apresentar a nossa versão da definição de um espaço-tempo assintoticamente plano. A definição que propomos constitui uma ligeira modificação das definições adotadas originalmente por Ashtekar \& Romano [10] e subseqüentemente por Perng [11]; comentaremos as diferenças logo abaixo. 
Definição 1.3 Dizemos que um espaço-tempo $(M, g)$ admite uma fronteira assintoticamente plana ou um bordo assintoticamente plano no infinito espacial se existe um completamento $(\hat{M}, \hat{g}, \Omega)$ de $(M, g)$ com as seguintes propriedades:

1. Numa vizinhança colar de $\partial \hat{M}$, a métrica não-física $\hat{g}$ e a métrica física g são relacionadas por um reescalonamento conforme anisotrópico, como nas equações (1.23) e (1.49), $\operatorname{com} s=1$ e $t=2$ :

$$
\begin{aligned}
& g_{a b}=q_{a b}+n_{a} n_{b} \quad, \quad \hat{g}_{a b}=\Omega^{2} q_{a b}+\Omega^{4} n_{a} n_{b} \\
& g^{a b}=q^{a b}+n^{a} n^{b} \quad, \quad \hat{g}^{a b}=\Omega^{-2} q^{a b}+\Omega^{-4} n^{a} n^{b}
\end{aligned}
$$

2. O tensor de Einstein físico satisfaz condições de decaimento ao infinito no sentido que os limites

$$
\begin{gathered}
\lim _{\Omega \rightarrow 0} \hat{n}^{a} \hat{n}^{b} G_{a b} \\
\lim _{\Omega \rightarrow 0} \Omega^{-1} \hat{P}_{a}^{c} \hat{n}^{d} G_{c d} \\
\lim _{\Omega \rightarrow 0} \Omega^{-2} \hat{P}_{a}^{c} \hat{P}_{b}^{d} G_{c d}
\end{gathered}
$$

existem e são suaves.

Dizemos ainda que o espaço-tempo $(M, g)$ é assintoticamente plano no infinito espacial se este completamento pode ser escolhido de modo que a fronteira $\partial \hat{M}$ é difeomorfa a $\mathbb{R} \times S^{2}$ e que é assintoticamente minkowskiano no infinito espacial se, além disso, a fronteira $\partial \hat{M}$ for geodesicamente completa na métrica induzida $\left.\hat{q}\right|_{\partial \hat{M}}$.

As diferenças principais entre nossa definição de uma fronteira assintoticamente plana no infinito espacial e as definições encontradas nos dois trabalhos já citados (onde tal fronteira é chamada de "asymptote at spatial infinity") são as seguintes:

- As condições de decaimento para o tensor de Einstein coincidem com as de [11] mas são mais fracas que as de [10]. O motivo é que as primeiras são adequadas enquanto que as últimas são fisicamente inaceitáveis. Para justificar esta afirmação, notamos que as condições corretas devem valer para todos os tensores de energia-momento conhecidos da física que descrevem fontes do campo gravitacional localizadas em regiões limitadas, e estes apresentam diversas taxas de decaimento, sendo que a mais lenta é a do campo eletrostático, com potencial de Coulomb $\sim 1 / r$, campo $\sim 1 / r^{2}$ e densidade de energia $\sim 1 / r^{4}$. Ocorre que, justamente para este caso, existe uma solução exata das equações de Einstein, estática e esfericamente simétrica: a 
solução de Reissner-Nordström, e como observado em [11] e demonstrado explicitamente no próximo capítulo, as condições de [10] não são satisfeitas neste caso. (Diga-se de passagem que os autores de [10] consideram como exemplo principal de toda a sua metodologia do completamento por reescalonamento conforme anisotrópico apenas a solução de Schwarzschild que, sendo uma solução de vácuo, é totalmente improdutiva para analisar a questão das condições de decaimento do tensor de energia-momento: assim, a inadequação da definição por eles proposta passou despercebida.) O que ainda falta é verificar se as condições de [11] também valem para a solução de Kerr-Newman.

- A definição adotada em [11] inclui duas restrições adicionais que nós omitimos na nossa definição e que têm o caráter de condições de normalização ou de "calibragem". Na notação usada aqui, são

$$
\hat{F}=1 \quad \text { em } \partial \hat{M}
$$

e

$$
\mathcal{L}_{\hat{F}^{1 / 2} \hat{n}}\left(\hat{F}^{-1} \hat{q}_{a b}\right)=0 \quad \text { em } \partial \hat{M}
$$

Optamos por não incluir estas restrições desde o início por motivos a serem discutidos logo adiante.

Para facilitar a comparação, apresentamos na tabela abaixo uma lista de correspondências entre objetos na notação de $[10,11]$ e na nossa notação, que acreditamos ser mais sistemática e portanto mais fácil de memorizar.

Na prática, a definição de um espaço-tempo assintoticamente plano ou assintoticamente minkowskiano não é fácil de se verificar, pois pode estar longe de ser óbvio como construir uma função $\Omega$ com as propriedades desejadas. (Por exemplo, pode acontecer que a escolha mais "natural" deixa de providenciar uma extensão suave, como mostra o exemplo da solução de Schwarzschild e de Reissner-Nordström apresentado no próximo capítulo.) Além disso, a função $\Omega$ está longe de ser única e portanto é preciso ter um critério para decidir quais funções levam a completamentos equivalentes. Nesta direção, note primeiro que se $\Omega$ e $\Omega^{\prime}$ são duas funções na mesma variedade $\hat{M}$ tais que $(\hat{M}, \hat{g}, \Omega)$ e $\left(\hat{M}, \hat{g}^{\prime}, \Omega^{\prime}\right)$ são completamentos do mesmo espaço-tempo físico $(M, g)$, conforme a Definição 1.3, então devemos ter $\Omega^{\prime}=\alpha \Omega$ com uma função suave $\alpha$ estritamente positiva em $\hat{M},{ }^{2}$ e como ambas as funções $\hat{F}$ e $\hat{F}^{\prime}$ e ambos os campos vetoriais normais $\hat{n}$ e $\hat{n}^{\prime}$

\footnotetext{
${ }^{2}$ Se $\alpha$ tivesse um zero no interior $M$ de $\hat{M}$, não valeria $\Omega^{\prime}>0$ em $M$, e se $\alpha$ tivesse um zero no bordo $\partial \hat{M}$ de $\hat{M}$, não valeria $d \Omega^{\prime} \neq 0$ em $\partial \hat{M}$.
} 


\begin{tabular}{|c|c|c|}
\hline \hline Objeto & Notação de $[10,11]$ & Notação neste trabalho \\
\hline \hline Espaço-tempo físico & $\hat{M}$ & $M$ \\
\hline Completamento & $M$ & $\partial \hat{M}$ \\
\hline Fronteira & $\mathcal{H}$ & $g_{a b}=q_{a b}+n_{a} n_{b}$ \\
\hline Métrica física & $\hat{g}_{a b}=\Omega^{-2} q_{a b}+l^{-1} \partial_{a} \Omega \partial_{b} \Omega$ & $\hat{g}_{a b}=\hat{q}_{a b}+\hat{n}_{a} \hat{n}_{b}$ \\
\hline Métrica auxiliar & $g_{a b}=q_{a b}+F^{-1} \partial_{a} \Omega \partial_{b} \Omega$ & $F$ \\
\hline Normalização física & $l$ & $\hat{F}$ \\
\hline Normalização auxiliar & $F$ & $q_{a b}$ \\
\hline Métrica física induzida & $\hat{q}_{a b}$ & $\hat{q}_{a b}$ \\
\hline Métrica auxiliar induzida & $q_{a b}$ & $n^{a}$ \\
\hline Vetor normal físico & $\hat{n}^{a}$ & $\hat{F}^{1 / 2} \hat{n}^{a}=\hat{g}^{a b} \partial_{b} \Omega$ \\
\hline Vetor normal auxiliar & $n^{a}$ & $K_{a b}$ \\
\hline $2^{\text {a forma fundamental física }}$ & $\hat{K}_{a b}$ & \\
\hline \hline
\end{tabular}

admitem extensões suaves ao bordo $\partial \hat{M}$ de $\hat{M}$, podemos usar a relação

$$
\begin{aligned}
\hat{F}^{1 / 2} \hat{n}^{\prime a} & =\hat{g}^{\prime a b} \partial_{b} \Omega^{\prime}=\Omega^{\prime-4} g^{a b} \partial_{b} \Omega^{\prime}=\alpha^{-4} \Omega^{-4} g^{a b}\left(\partial_{b} \alpha \Omega+\alpha \partial_{b} \Omega\right) \\
& =\alpha^{-4} \Omega^{-3} g^{a b} \partial_{b} \alpha+\alpha^{-3} \Omega^{-4} g^{a b} \partial_{b} \Omega \\
& =\alpha^{-4} \Omega^{-3}\left(\Omega^{2} \hat{q}^{a b}+\Omega^{4} \hat{n}^{a} \hat{n}^{b}\right) \partial_{b} \alpha+\alpha^{-3} \hat{g}^{a b} \partial_{b} \Omega \\
& =\alpha^{-4} \Omega^{-1} \hat{q}^{a b} \partial_{b} \alpha+\alpha^{-4} \Omega \hat{n}^{a} \hat{n}^{b} \partial_{b} \alpha+\alpha^{-3} \hat{F}^{1 / 2} \hat{n}^{a}
\end{aligned}
$$

para concluir que $\alpha$ deve ser constante no bordo $\partial \hat{M}$ de $\hat{M}$. Por outro lado, segundo a equação (1.69), temos

$$
\hat{P}_{a}^{c} \hat{n}^{d} G_{c d}=\hat{P}_{a}^{c} \hat{n}^{d} R_{c d}=\hat{P}_{a}^{c} \hat{n}^{d} \hat{R}_{c d}+\Omega^{-1} \hat{F}^{-1 / 2} \hat{P}_{a}^{c}\left(\partial_{c} \hat{F}\right)
$$

mas conforme a condição (1.74), esta expressão se anula no bordo $\partial \hat{M}$ de $\hat{M}$, o que só é possível se $\hat{F}$ é constante no bordo $\partial \hat{M}$ de $\hat{M}$. Portanto, multiplicando $\Omega$ por 
uma constante apropriada, podemos garantir a validade da condição de normalização (1.76) e, ao mesmo tempo, concluir que isso determina a função $\Omega$ completamente até primeira ordem, ou seja: se $\Omega$ e $\Omega^{\prime}$ são duas funções na mesma variedade $\hat{M}$ tais que $(\hat{M}, \hat{g}, \Omega)$ e $\left(\hat{M}, \hat{g}^{\prime}, \Omega^{\prime}\right)$ são completamentos do mesmo espaço-tempo físico $(M, g)$ e tais que $\hat{F}=1$ e $\hat{F}^{\prime}=1$ no bordo $\partial \hat{M}$ de $\hat{M}$, então existe uma função suave $\omega$ em $\hat{M}$ tal que $\Omega^{\prime}=\Omega+\omega \Omega^{2}$. Conforme argumentado em [10], os completamentos devem ser considerados equivalentes se as duas funções coincidem não apenas até segunda ordem mas mesmo até terceira ordem, ou seja, se $\omega$ também se anula em $\partial \hat{M}$, e portanto a restrição de $\omega$ ao bordo $\partial \hat{M}$ de $\hat{M}$ descreve o que os autores de [10] chamam de "estrutura de segunda ordem", que desempenha um papel importante na definição de quantidades conservadas associadas a um espaço-tempo assintoticamente plano, tais como energia-momento (inclusive a massa ADM) e momento angular, em termos de dados assintóticos: nós não pretendemos perseguir esta linha de raciocínio aqui.

Além da condição de normalização (1.76) provindo da condição de decaimento (1.74) das componentes mistas do tensor de Einstein, há outra conclusão importante que pode ser retirada da condição de decaimento (1.73) das suas componentes puramente normais e, principalmente, da condição de decaimento (1.75) das suas componentes puramente tangenciais. Por exemplo, multiplicando a equação (1.70) por $\Omega^{-2}$ e tomando o limite $\Omega \rightarrow 0$, obtemos

$$
-\frac{1}{2} \hat{\mathcal{R}}+3 \hat{F}=0 \quad \text { em } \partial \hat{M}
$$

enquanto que tomando o limite $\Omega \rightarrow 0$ na equação (1.71), obtemos

$$
\begin{gathered}
\hat{\mathcal{R}}_{a b}-\frac{1}{2} \hat{\mathcal{R}} \hat{q}_{a b}+\hat{F} \hat{q}_{a b}-\hat{F}^{1 / 2}\left(\hat{\mathcal{D}}_{a} \hat{\mathcal{D}}_{b} \hat{F}^{-1 / 2}\right)+\hat{q}^{c d} \hat{F}^{1 / 2}\left(\hat{\mathcal{D}}_{c} \hat{\mathcal{D}}_{d} \hat{F}^{-1 / 2}\right) \hat{q}_{a b}=0 \\
\text { em } \partial \hat{M} .
\end{gathered}
$$

Combinando estas equações com a equação (1.76) concluímos que

$$
\begin{gathered}
\hat{F}=1, \quad \hat{\mathcal{D}}_{a} \hat{F}=0, \hat{\mathcal{D}}_{a} \hat{\mathcal{D}}_{b} \hat{F}=0 \quad \text { em } \partial \hat{M} \\
\hat{\mathcal{R}}_{a b}=2 \hat{q}_{a b}, \hat{\mathcal{R}}=6 \quad \text { em } \partial \hat{M}
\end{gathered}
$$

Em particular, isso significa que o bordo $\partial \hat{M}$ de $\hat{M}$, com a métrica induzida pela métrica não-física $\hat{q}$, é um espaço de curvatura constante não-nula.

Existe ainda a possibilidade de estudar as restrições impostas sobre a geometria do completamento $(\hat{M}, \hat{g}, \Omega)$ próximo ao bordo $\partial \hat{M}$ de $\hat{M}$ que resultam da análise das condições (1.73), (1.74) e (1.75) na próxima ordem da expansão em termos de potências de $\Omega$. Isso leva a condições relacionando as derivadas de Lie das quantidades que aparecem na equação (1.78) ao longo do campo vetorial normal $\hat{n}$ com outras quantidades, 
principalmente a segunda forma fundamental $\hat{K}_{a b}$ no bordo $\partial \hat{M}$ de $\hat{M}$, que descreve a parte extrínsica da curvatura do mergulho de $\partial \hat{M}$ em $\hat{M}$. Também não pretendemos perseguir esta linha de raciocínio aqui, pois ela já foi desenvolvida de forma sistemática em [11].

Resta apenas comentar o papel da condição de normalização (1.77) que aparentemente não pode ser deduzida desta forma, mas constitui uma restrição não-trivial sobre os completamentos $(\hat{M}, \hat{g}, \Omega)$ permitidos. Segundo [10] e [11], essa restrição é necessária para poder definir não apenas a energia e o momento mas também o momento angular do espaço-tempo $(M, g)$. Não é claro se essa condição pode sempre ser imposta sem perda de generalidade, através de uma mudança apropriada da função $\Omega$ dentro de sua classe de equivalência, ou se ela constitui uma hipótese adicional e, neste caso, qual seria o seu significado físico. Contudo, parece não haver contra-exemplos, pois conforme argumentado em [11], todos os espaços-tempos estacionários que são assintoticamente planos no sentido tradicional [1, 5] são assintoticamente planos no sentido da definição de [11], a qual inclui a condição de normalização (1.77). 
Capítulo 2

\section{Soluções de Schwarzschild e de Reissner-Nordström}

A nossa meta principal neste capítulo é mostrar como as soluções exatas conhecidas das equações de Einstein estáticas e com simetria esférica, as de Schwarzschild e de ReissnerNordström, se encaixam na definição de um espaço-tempo assintoticamente plano desenvolvida no capítulo anterior, sendo que a primeira é uma solução de vácuo, com tensor de energia-momento identicamente nulo, enquanto que a segunda é caracterizada por um tensor de energia-momento com o decaimento ao infinito mais lento conhecido na física, pois é gerado por um campo de longo alcance, o campo eletrostático de Coulomb. Uma definição do conceito de espaço-tempo assintoticamente plano que permite incorporar este exemplo tem boas chances de abranger todas as situações de relevância física. (Outro teste extremamente relevante seria investigar as soluções de Kerr-Newman, que são apenas estacionárias e apresentam apenas simetria axial, descrevendo espaços-tempos com momento angular não-nulo, mas não trataremos deste caso aqui.)

\subsection{Soluções exatas com simetria esférica}

Em coordenadas esféricas padrão $(t, r, \theta, \varphi)$, a métrica de um espaço-tempo estático e esfericamente simétrico é dada por

$$
d s^{2}=-\exp (2 a(r)) c^{2} d t^{2}+\exp (2 b(r)) d r^{2}+r^{2}\left(d \theta^{2}+\sin ^{2} \theta d \varphi^{2}\right),
$$

com duas funções $a$ e $b$ da variável radial $r^{1}$ cuja determinação remonta à solução das equações de Einstein. Para tanto, é mais conveniente trabalhar no referencial ortonormal

\footnotetext{
${ }^{1}$ De maneira invariante, a variável $r$ pode, em situações com simetria esférica, ser caracterizada como o único parâmetro rotulando as órbitas sob o grupo $S O(3)$ como esferas cuja área, medida pela métrica induzida, vale $4 \pi r^{2}$; esta condiçao fixa o coeficiente do termo $d \theta^{2}+\sin ^{2} \theta d \varphi^{2}$ como sendo igual a $r^{2}$.
} 
$\left\{e_{0}, e_{1}, e_{2}, e_{3}\right\}$ de campos vetoriais definido por

$$
\begin{gathered}
e_{0}=\exp (-a(r)) \frac{1}{c} \frac{\partial}{\partial t} \quad, \quad e_{2}=\frac{1}{r} \frac{\partial}{\partial \theta} \\
e_{1}=\exp (-b(r)) \frac{\partial}{\partial r} \quad, \quad e_{3}=\frac{1}{r \sin \theta} \frac{\partial}{\partial \varphi}
\end{gathered}
$$

e no referencial ortonormal dual $\left\{e^{0}, e^{1}, e^{2}, e^{3}\right\}$ de 1-formas dado por

$$
\begin{gathered}
e^{0}=\exp (a(r)) c d t, \quad e^{2}=r d \theta \\
e^{1}=\exp (b(r)) d r, \quad e^{3}=r \sin \theta d \varphi
\end{gathered}
$$

o que, após um cálculo padrão, leva às seguintes expressões para as componentes do tensor de Einstein neste referencial:

$$
\begin{gathered}
G_{00}=\frac{2 b^{\prime}}{r} \exp (-2 b)+\frac{1-\exp (-2 b)}{r^{2}}, \\
G_{11}=\frac{2 a^{\prime}}{r} \exp (-2 b)-\frac{1-\exp (-2 b)}{r^{2}}, \\
G_{22}=-\left(a^{\prime} b^{\prime}-a^{\prime \prime}-\left(a^{\prime}\right)^{2}-\frac{a^{\prime}-b^{\prime}}{r}\right) \exp (-2 b)=G_{33} .
\end{gathered}
$$

No caso da solução de Schwarzschild, este é identicamente nulo, mas no caso da solução de Reissner-Nordström, há como campo de matéria um campo eletrostático com potencial dado pela 1-forma

$$
A=f(r) c d t=f(r) \exp (-a(r)) e^{0}
$$

e tensor de campo dado pela 2-forma

$$
F=-f^{\prime}(r) c d t \wedge d r=-f^{\prime}(r) \exp (-(a(r)+b(r))) e^{0} \wedge e^{1},
$$

com uma terceira função $f$ da variável radial $r$, o que leva às seguintes expressões para as componentes do tensor de energia-momento neste referencial:

$$
\begin{gathered}
T_{00}=\frac{1}{2 \mu_{0}} f^{\prime 2} \exp (-2(a+b)), \\
T_{11}=-\frac{1}{2 \mu_{0}} f^{\prime 2} \exp (-2(a+b)), \\
T_{22}=\frac{1}{2 \mu_{0}} f^{\prime 2} \exp (-2(a+b))=T_{33} .
\end{gathered}
$$

Aqui, $\mu_{0}$ denota a constante magnética ou permeabilidade do vácuo. ${ }^{2}$

\footnotetext{
${ }^{2}$ Veja os comentários referentes a sistemas de unidades no fim desta seção.
} 
As funções $a, b$ e $f$ são determinadas pela solução das equações de Einstein-Maxwell, sendo que as equações de Einstein,

$$
G_{a b}=\frac{8 \pi \gamma}{c^{4}} T_{a b}
$$

onde $\gamma$ denota a constante gravitacional de Newton, ${ }^{2}$ providenciam duas equações independentes e as equações de Maxwell (no vácuo),

$$
g^{a b} \nabla_{a} F_{b c}=0
$$

providenciam uma terceira:

$$
f^{\prime \prime}+\frac{2}{r} f^{\prime}-f^{\prime}\left(a^{\prime}+b^{\prime}\right)=0 .
$$

Impondo as condições assintóticas

$$
\lim _{r \rightarrow \infty} a(r)=0, \lim _{r \rightarrow \infty} b(r)=0, \lim _{r \rightarrow \infty} f(r)=0
$$

podemos prosseguir da seguinte maneira. Em primeiro lugar, simplificamos o sistema somando as componentes 00 e 11 das equações de Einstein, o que implica que

$$
a+b=0
$$

Em segundo lugar, usamos este fato para resolver a equação de Maxwell, o que leva à conclusão de que $f(r)$ é proporcional a $1 / r$ (potencial de Coulomb). Em terceiro lugar, inserimos este resultado na componente 00 ou 11 das equações de Einstein para deduzir uma equação diferencial para a função $r \exp (2 a(r))$ que é fácil de resolver. Como resultado final, obtemos a expressão tradicional para a métrica de Reissner-Nordström,

$$
d s^{2}=-\sigma(r) c^{2} d t^{2}+\sigma(r)^{-1} d r^{2}+r^{2}\left(d \theta^{2}+\sin ^{2} \theta d \varphi^{2}\right)
$$

com tensor de Einstein dado por

$$
G=\frac{r_{Q}^{2}}{4 r^{4}}\left(\sigma(r) c^{2} d t^{2}-\sigma(r)^{-1} d r^{2}+r^{2}\left(d \theta^{2}+\sin ^{2} \theta d \varphi^{2}\right)\right)
$$

que deve ser complementada pelas expressões para o potencial eletrostático e o campo eletrostático de Coulomb,

$$
A=\frac{Q}{4 \pi \epsilon_{0} \kappa} \frac{1}{r} d t \quad, \quad F=\frac{Q}{4 \pi \epsilon_{0} \kappa} \frac{1}{r^{2}} d t \wedge d r
$$


com o tensor de energia-momento padrão,

$$
T=\frac{Q^{2}}{32 \pi^{2} \epsilon_{0}} \frac{1}{r^{4}}\left(\sigma(r) c^{2} d t^{2}-\sigma(r)^{-1} d r^{2}+r^{2}\left(d \theta^{2}+\sin ^{2} \theta d \varphi^{2}\right)\right),
$$

onde $\epsilon_{0}$ denota a constante dielétrica do vácuo, ${ }^{2}$ e onde usamos a abreviação

$$
\sigma(r)=1-\frac{r_{M}}{r}+\frac{r_{Q}^{2}}{4 r^{2}}
$$

Aqui, $r_{M}$ e $r_{Q}$ são dois comprimentos característicos do problema definidos, respectivamente, pela massa $M$ e pela carga $Q$ localizadas na origem $r=0$ deste sistema de coordenadas, onde tanto a métrica como o campo eletromagnético são singulares: um deles é o tradicional raio de Schwarzschild

$$
r_{M}=\frac{2 \gamma M}{c^{2}}
$$

enquanto que o outro é definido por

$$
r_{Q}=\sqrt{\frac{\gamma}{\pi \epsilon_{0}}} \frac{Q}{c^{2}} .
$$

Em situações de relevância física, temos $r_{Q}<r_{M}$ (e na verdade, $r_{Q} \ll r_{M}$ ), de modo que a função $\sigma$ tem dois zeros simples nos pontos

$$
r_{ \pm}=\frac{r_{M}}{2}\left(1 \pm \sqrt{1-r_{Q}^{2} / r_{M}^{2}}\right)
$$

sendo positiva na região exterior definida por $r_{+}<r<\infty$ e na região interior definida por $0<r<r_{-}$, mas negativa na região intermediária definida por $r_{-}<r<r_{+}$: essas regiões são separadas por um horizonte externo localizado em $r=r_{+}$e um horizonte interno localizado em $r=r_{-}$. A seguir, consideraremos apenas a região exterior, fora do horizonte externo, onde (ao contrário da região intermediária) as variáveis $r$ e $t$ mantêm seus papéis tradicionais, com $\partial / \partial t$ tipo-tempo e $\partial / \partial r$ tipo-espaço.

Obviamente, a solução de Reissner-Nordström contém como caso especial a solução de Schwarzschild: basta escolher $Q=0$.

Quanto à escolha das constantes nas fórmulas acima, notamos que estas se aplicam em qualquer sistema de unidades, sendo que as constantes $\epsilon_{0}$ (a constante dielétrica do vácuo), $\mu_{0}$ (a constante magnética ou permeabilidade do vácuo) e a constante $\kappa$ que aparece na lei de indução de Faraday na forma

$$
\boldsymbol{\nabla} \times \boldsymbol{E}=-\kappa \frac{\partial \boldsymbol{B}}{\partial t}
$$

e que assume o valor 1 (em sistemas de unidades assimétricos, principalmente o SI) ou 1/c (em sistemas de unidades simétricos, como os de Gauss ou Heaviside), são relacionadas com a velocidade da luz $c$ pela fórmula universal $\epsilon_{0} \mu_{0}(\kappa c)^{2}=1$. 


\subsection{Completamento conforme anisotrópico}

Desejamos agora aplicar a metodologia do reescalonamento conforme anisotrópico desenvolvida no primeiro capítulo à solução de Reissner-Nordström, que contém a de Schwarzschild como caso especial. Para tanto, adotaremos o mesmo procedimento de [10], introduzindo coordenadas hiperbólicas $(\tau, \rho, \theta, \varphi)$ definidas por $^{2}$

$$
\begin{aligned}
& c t=\rho \sinh \tau \\
& r=\rho \cosh \tau
\end{aligned}
$$

de modo que, reciprocamente,

$$
\begin{gathered}
\rho=\sqrt{r^{2}-c^{2} t^{2}} \\
\tau=\tanh ^{-1}(c t / r)
\end{gathered}
$$

e em seguida passamos a coordenadas $(\Omega, \tau, \theta, \varphi)$ com

$$
\Omega=\frac{1}{\rho} .
$$

Então o tensor métrico de Reissner-Nordström e o seu tensor de Einstein são dados pelas expressões

$$
\begin{aligned}
d s^{2}= & -\rho^{2}\left(\sigma \cosh ^{2} \tau-\sigma^{-1} \sinh ^{2} \tau\right) d \tau^{2} \\
& +2 \rho \sinh \tau \cosh \tau\left(\sigma^{-1}-\sigma\right) d \rho d \tau \\
& +\left(\sigma^{-1} \cosh ^{2} \tau-\sigma \sinh ^{2} \tau\right) d \rho^{2} \\
& +\rho^{2} \cosh ^{2} \tau\left(d \theta^{2}+\sin ^{2} \theta d \varphi^{2}\right)
\end{aligned}
$$

e

$$
\begin{aligned}
G=\frac{r_{Q}^{2}}{4(\rho \cosh \tau)^{4}}( & \rho^{2}\left(\sigma \cosh ^{2} \tau-\sigma^{-1} \sinh ^{2} \tau\right) d \tau^{2} \\
& -2 \rho \sinh \tau \cosh \tau\left(\sigma^{-1}-\sigma\right) d \rho d \tau \\
& -\left(\sigma^{-1} \cosh ^{2} \tau-\sigma \sinh ^{2} \tau\right) d \rho^{2} \\
& \left.+\rho^{2} \cosh ^{2} \tau\left(d \theta^{2}+\sin ^{2} \theta d \varphi^{2}\right)\right)
\end{aligned}
$$

com

$$
\sigma(\rho)=1-\frac{r_{M}}{\rho \cosh \tau}+\frac{r_{Q}^{2}}{4(\rho \cosh \tau)^{2}},
$$

\footnotetext{
${ }^{2}$ Usamos a letra $\tau$, em vez de $\chi$, para sugerir que se trata de uma variável tipo tempo.
} 
$\mathrm{Ou}$

$$
\begin{aligned}
d s^{2}= & \Omega^{-4}\left(\sigma^{-1} \cosh ^{2} \tau-\sigma \sinh ^{2} \tau\right) d \Omega^{2} \\
& -2 \Omega^{-3} \sinh \tau \cosh \tau\left(\sigma^{-1}-\sigma\right) d \Omega d \tau \\
& +\Omega^{-2}\left(-\left(\sigma \cosh ^{2} \tau-\sigma^{-1} \sinh ^{2} \tau\right) d \tau^{2}\right. \\
& \left.\quad+\cosh ^{2} \tau\left(d \theta^{2}+\sin ^{2} \theta d \varphi^{2}\right)\right)
\end{aligned}
$$

e

$$
\begin{aligned}
& G=\frac{r_{Q}^{2} \Omega^{4}}{4 \cosh ^{4} \tau}\left(-\Omega^{-4}\left(\sigma^{-1} \cosh ^{2} \tau-\sigma \sinh ^{2} \tau\right) d \Omega^{2}\right. \\
& +2 \Omega^{-3} \sinh \tau \cosh \tau\left(\sigma^{-1}-\sigma\right) d \Omega d \tau \\
& +\Omega^{-2}\left(\left(\sigma \cosh ^{2} \tau-\sigma^{-1} \sinh ^{2} \tau\right) d \tau^{2}\right. \\
& \left.\left.+\cosh ^{2} \tau\left(d \theta^{2}+\sin ^{2} \theta d \varphi^{2}\right)\right)\right),
\end{aligned}
$$

com

$$
\sigma(\Omega)=1-\frac{r_{M} \Omega}{\cosh \tau}+\frac{r_{Q}^{2} \Omega^{2}}{4 \cosh ^{2} \tau}
$$

Calculando a matriz inversa $g^{a b}$ da matriz $g_{a b}$ representada pela equação (2.26) e usando que $F=g^{\Omega \Omega}$ e

$$
n^{b}=F^{-1 / 2} d \Omega,
$$

assim como $q_{a b}=g_{a b}-n_{a} n_{b}$, obtemos por um cálculo elementar

$$
F=\Omega^{4}\left(\sigma \cosh ^{2} \tau-\sigma^{-1} \sinh ^{2} \tau\right)
$$

$\mathrm{e}$

$$
n=F^{-1 / 2}\left(F \frac{\partial}{\partial \Omega}-\Omega^{3} \sinh \tau \cosh \tau\left(\sigma^{-1}-\sigma\right) \frac{\partial}{\partial \tau}\right)
$$

assim como

$$
\begin{aligned}
q= & -F^{-1} \sinh ^{2} \tau \cosh ^{2} \tau\left(\sigma^{-1}-\sigma\right)^{2} d \Omega^{2} \\
& -2 \Omega^{-3} \sinh \tau \cosh \tau\left(\sigma^{-1}-\sigma\right) d \Omega d \tau \\
& +\Omega^{-2}\left(-\Omega^{-4} F d \tau^{2}+\cosh ^{2} \tau\left(d \theta^{2}+\sin ^{2} \theta d \varphi^{2}\right)\right)
\end{aligned}
$$

Reescalonando $q$ e $n$ com a segunda potência de $\Omega$, obtemos

$$
\hat{F}=\sigma \cosh ^{2} \tau-\sigma^{-1} \sinh ^{2} \tau
$$

e

$$
\hat{n}=\hat{F}^{-1 / 2}\left(\hat{F} \frac{\partial}{\partial \Omega}-\Omega^{-1} \sinh \tau \cosh \tau\left(\sigma^{-1}-\sigma\right) \frac{\partial}{\partial \tau}\right)
$$


assim como

$$
\begin{aligned}
\hat{q}= & -\Omega^{-2} \hat{F}^{-1} \sinh ^{2} \tau \cosh ^{2} \tau\left(\sigma^{-1}-\sigma\right)^{2} d \Omega^{2} \\
& -2 \Omega^{-1} \sinh \tau \cosh \tau\left(\sigma^{-1}-\sigma\right) d \Omega d \tau \\
& -\hat{F} d \tau^{2}+\cosh ^{2} \tau\left(d \theta^{2}+\sin ^{2} \theta d \varphi^{2}\right) .
\end{aligned}
$$

Notamos que potências negativas de $\Omega$ sempre aparecem como potências da combinação $\Omega^{-1} \cosh \tau\left(\sigma^{-1}-\sigma\right)$ e que esta é inócua, pois como função de $\Omega$ (ou melhor, de $\Omega / \cosh \tau=1 / r)$ a função $\sigma$ satisfaz $\sigma(0)=1$ e assim a expressão

$$
\Omega^{-1} \cosh \tau \frac{1-\sigma^{2}}{\sigma}
$$

é uma função racional de $\Omega / \cosh \tau=1 / r$ e não-singular na origem.

Inspeção direta das equações (2.27) e (2.33) revela agora que o tensor de Einstein da métrica de Reissner-Nordström satisfaz as condições de decaimento (1.73), (1.74) e (1.75) e que vale a condição de normalização (1.76), mas que não satisfaz as condições de decaimento

$$
\lim _{\Omega \rightarrow 0} \Omega^{-1} G_{a b}=0
$$

(uniformemente para todas as componentes) exigidas em [10].

O maior inconveniente do cálculo que acabamos de apresentar consiste no fato de que no sistema de coordenadas $(\Omega, \tau, \theta, \varphi)$ utilizado acima, o tensor métrico não é diagonal. Note que isso não constitui um problema maior ou de princípio, mas apenas uma complicação que à primeira vista parece desnecessária, uma vez que a solução de ReissnerNordström é estática e esfericamente simétrica, o que deveria nos permitir trabalhar com coordenadas em que a métrica é diagonal, o que simplificaria os cálculos. Isso é de fato possível se substituírmos a variável radial padrão $r$ pela coordenada tartaruga $r_{*}$ de Regge-Wheeler, que é definida como a integral da componente radial da métrica relativo à variável radial $r$, ou seja, como a solução da equação

$$
\frac{d r_{*}}{d r}=\sigma(r)^{-1}
$$

Explicitamente, podemos usar que

$$
\begin{aligned}
\sigma(r)^{-1} & =\frac{r^{2}}{\left(r-r_{+}\right)\left(r-r_{-}\right)}=\frac{1}{r_{+}-r_{-}}\left(\frac{r^{2}}{r-r_{+}}-\frac{r^{2}}{r-r_{-}}\right) \\
& =\frac{1}{r_{+}-r_{-}}\left(\frac{r_{+} r}{r-r_{+}}-\frac{r_{-} r}{r-r_{-}}\right) \\
& =1+\frac{1}{r_{+}-r_{-}}\left(\frac{r_{+}^{2}}{r-r_{+}}-\frac{r_{-}^{2}}{r-r_{-}}\right),
\end{aligned}
$$


para integrar esta equação diretamente:

$$
r_{*}=r+\frac{r_{+}^{2}}{r_{+}-r_{-}} \ln \left(\frac{r}{r_{+}}-1\right)-\frac{r_{-}^{2}}{r_{+}-r_{-}} \ln \left(\frac{r}{r_{-}}-1\right) .
$$

Observe que esta fórmula define um difeomorfismo

$$
\begin{aligned}
& ] r_{+}, \infty[\longrightarrow \mathbb{R} \\
& r \longmapsto r_{*}
\end{aligned}
$$

Poderíamos então efetuar a mesma transformação para coordenadas hiperbólicas $(\tau, \rho, \theta, \varphi)$ como acima, só que com $r_{*}$ no lugar de $r$,

$$
\begin{aligned}
& c t=\rho \sinh \tau \\
& r_{*}=\rho \cosh \tau
\end{aligned},
$$

de modo que, reciprocamente,

$$
\begin{gathered}
\rho=\sqrt{r_{*}^{2}-c^{2} t^{2}} \\
\tau=\tanh ^{-1}\left(c t / r_{*}\right)
\end{gathered}
$$

e definir

$$
\Omega=\frac{1}{\rho} .
$$

Um simples cálculo mostra então que, no sistema de coordenadas $(\Omega, \tau, \theta, \varphi)$ assim definido, o tensor métrico de Reissner-Nordström e o seu tensor de Einstein são dados por

$$
d s^{2}=\Omega^{-4} \sigma(r) d \Omega^{2}+\Omega^{-2}\left(-\sigma(r) d \tau^{2}+\frac{r^{2}}{r_{*}^{2}} \cosh ^{2} \tau\left(d \theta^{2}+\sin ^{2} \theta d \varphi^{2}\right)\right)
$$

e

$$
G=\frac{r_{Q}^{2}}{4 r^{4}}\left(-\Omega^{-4} \sigma(r) d \Omega^{2}+\Omega^{-2}\left(\sigma(r) d \tau^{2}+\frac{r^{2}}{r_{*}^{2}} \cosh ^{2} \tau\left(d \theta^{2}+\sin ^{2} \theta d \varphi^{2}\right)\right)\right) .
$$

Infelizmente, não existe uma expressão elementar que permita inverter a relação (2.38) e obter $r$ como função explícita de $r_{*}$ ou de $\tau$ e $\rho$ ou de $\tau$ e $\Omega$, respectivamente. De qualquer forma, temos

$$
\Omega \rightarrow 0 \Longrightarrow r_{*} \rightarrow \infty \Longleftrightarrow r \rightarrow \infty
$$


e, neste limite,

$$
\frac{r_{*}}{r} \rightarrow 1 \quad, \quad \frac{d r_{*}}{d r} \rightarrow 1
$$

o que sugere introduzir uma métrica não física por

$$
d \hat{s}^{2}=\sigma(r) d \Omega^{2}-\sigma(r) d \tau^{2}+\frac{r^{2}}{r_{*}^{2}} \cosh ^{2} \tau\left(d \theta^{2}+\sin ^{2} \theta d \varphi^{2}\right) .
$$

O problema principal deste procedimento é que no limite (2.45), esta métrica é contínua mas não é suave, pois as primeiras derivadas das suas componentes $\hat{g}_{\theta \theta}$ e $\hat{g}_{\varphi \varphi}$ divergem logaritmicamente neste limite. De fato, como para qualquer função $\phi$ só de $r$, temos

$$
\frac{\partial \phi}{\partial \Omega}=\frac{d \rho}{d \Omega} \frac{\partial r_{*}}{\partial \rho} \frac{d r}{d r_{*}} \frac{d \phi}{d r}=-\Omega^{-2} \cosh \tau \sigma \frac{d \phi}{d r}
$$

$\mathrm{e}$

$$
\begin{aligned}
& \frac{d}{d r}\left(\frac{r^{2}}{r_{*}^{2}}\right)=-2\left(\frac{r_{*}}{r}\right)^{-3} \frac{d}{d r}\left(\frac{r_{*}}{r}\right) \\
&=-2\left(\frac{r_{*}}{r}\right)^{-3}\left(\frac{r_{+}^{2}}{r_{+}-r_{-}} \frac{d}{d r}\left(\frac{1}{r} \ln \left(\frac{r}{r_{+}}-1\right)\right)-\frac{r_{-}^{2}}{r_{+}-r_{-}} \frac{d}{d r}\left(\frac{1}{r} \ln \left(\frac{r}{r_{-}}-1\right)\right)\right) \\
&=-2\left(\frac{r_{*}}{r}\right)^{-3}\left(\frac{r_{+}^{2}}{r_{+}-r_{-}} \frac{1}{r\left(r-r_{+}\right)}-\frac{r_{+}^{2}}{r_{+}-r_{-}} \frac{1}{r^{2}} \ln \left(\frac{r}{r_{+}}-1\right)\right. \\
&\left.\quad-\frac{r_{-}^{2}}{r_{+}-r_{-}} \frac{1}{r\left(r-r_{-}\right)}+\frac{r_{-}^{2}}{r_{+}-r_{-}} \frac{1}{r^{2}} \ln \left(\frac{r}{r_{-}}-1\right)\right),
\end{aligned}
$$

vale

$$
\begin{aligned}
\frac{\partial}{\partial \Omega}\left(\frac{r^{2}}{r_{*}^{2}}\right)=\frac{2 \sigma}{\cosh \tau} \frac{r_{*}}{r} & \left(\frac{r_{+}^{2}}{r_{+}-r_{-}} \frac{r}{r-r_{+}}-\frac{r_{+}^{2}}{r_{+}-r_{-}} \ln \left(\frac{r}{r_{+}}-1\right)\right. \\
& \left.-\frac{r_{-}^{2}}{r_{+}-r_{-}} \frac{r}{r-r_{-}}+\frac{r_{-}^{2}}{r_{+}-r_{-}} \ln \left(\frac{r}{r_{-}}-1\right)\right) .
\end{aligned}
$$

Portanto, com esta escolha de coordenadas e da função $\Omega$, ambas as métricas (a física e a não-física) são diagonais, mas a métrica não-física deixa de ser suave no bordo e portanto o formalismo desenvolvido no primeiro capítulo não é diretamente aplicável. Assim, nos vemos forçados a abandonar este sistema de coordenadas e voltar ao sistema de coordenadas introduzido anteriormente, apesar do inconveniente de ter que trabalhar com métricas não-diagonais. 



\section{Conclusão}

Neste trabalho, iniciamos uma abordagem sistemática do problema de descrever a estrutura assintótica de variedades lorentzianas no infinito espacial por meio da introdução de uma métrica auxiliar relacionada com a métrica física do espaço-tempo por um "reescalonamento conforme anisotrópico". Trata-se de uma noção que já aparece nos trabalhos de Ashtekar \& Romano [10] e Perng [11], porém apenas de forma implícita, e que generaliza o reescalonamento conforme usual (que chamamos de "isotrópico") já introduzido por Hermann Weyl e usado por Roger Penrose para descrever a estrutura assintótica de variedades lorentzianas no infinito tipo-luz. Mostramos como este método permite controlar, de maneira natural, o comportamento assintótico do tensor métrico e de outros campos físicos no infinito espacial, o que é essencial para entender-se a estrutura de espaços-tempos assintoticamente planos. Como uma das primeiras conseqüências, chegamos à conclusão de que o bordo de tais espaços-tempos é um espaço (tridimensional e lorentziano) de curvatura constante. Também analisamos os exemplos não-triviais mais simples, as soluções de Schwarzschild e de Reissner-Nordström, e mostramos como se enquadram na nossa definição de um espaço-tempo assintoticamente plano. Uma tarefa que ainda está em aberto seria estender esta análise aos demais exemplos conhecidos, i.e., às soluções de Kerr e de Kerr-Newman.

Por outro lado, já existe na literatura toda uma teoria geral de espaços-tempos assintoticamente planos, cujo desenvolvimento se iniciou nos anos $60 \mathrm{com}$ a definição da massa ADM [1] e de outras quantidades conservadas (energia-momento, momento angular etc). É notável como esta teoria progrediu apesar da ausência de uma definição clara e simples do conceito de um espaço-tempo assintoticamente plano: as várias versões desta definição propostas até recentemente são todas tecnicamente complicadas e pouco transparentes. Sendo assim, coloca-se a tarefa de reformular as definições e os resultados principais da teoria dentro da nova metodologia aqui proposta, o que envolve (pelo menos) os seguintes problemas: 
- Analisar a questão de equivalência entre completamentos obtidos por diferentes funções de reescalonamento;

- Completar a análise das conseqüências das condições de fronteira impostas sobre o tensor de curvatura, o que requer:

- complementar a relação entre os tensores de Einstein, ou Ricci, com uma relação análoga entre os tensores de Weyl, e analisar as suas conseqüências quando se efetua uma expansão em potências da função de reescalonamento;

- estender a análise da expansão das condições de fronteira impostas sobre o tensor de curvatura em potências da função de reescalonamento a ordens superiores;

- Reformular as noções de "campos físicos assintoticamente regulares" e de seus "resíduos" empregadas por Perng [11] como condições de regularidade no bordo usando a métrica auxiliar.

Observamos que todas estas questões já foram abordadas em $[10,11]$, porém separandose sempre as componentes tangenciais das componentes normais, ou seja, sem fazer uso sistemático da métrica auxiliar obtida pelo reescalonamento conforme anisotrópico. 


\section{Bibliografia}

[1] R. Arnowitt, S. Deser \& C. Misner: Coordinate Invariance and Energy Expressions in General Relativity, Phys. Rev. 122 (1961) 997-1006.

[2] R. Penrose: Asymptotic Properties of Fields and Space-Times, Phys. Rev. Lett. 10 (1963) 66-68, Zero Rest-Mass Fields Including Gravitation: Asymptotic Behaviour, Proc. R. Soc. Lond. A 284 (1965) 159-203.

[3] S.W. Hawking \& G.F.R. Ellis: The Large Scale Structure of Space-Time, Cambridge University Press, Cambridge 1973.

[4] R.M. Wald: General Relativity, University of Chicago Press, Chicago 1984.

[5] R. Geroch: Structure of the Gravitational Field at Spatial Infinity, J. Math. Phys. 13 (1972) 956-968.

[6] A. Ashtekar \& R.O. Hansen: A Unified Treatment of Null and Spatial Infinity in General Relativity I. Universal Structure, Asymptotic Symmetries and Conserved Quantities at Spatial Infinity, J. Math. Phys. 19 (1978) 1542-1566.

[7] S. Persides: Structure of the Gravitational Field at Spatial Infinity. I. Asymptotically Euclidean Spaces, J. Math. Phys. 21 (1980) 135-142, Structure of the Gravitational Field at Spatial Infinity. II. Asymptotically Minkowskian Space-Times, J. Math. Phys. 21 (1980) 142-151.

[8] P. Sommers: The Geometry of the Gravitational Field at Spacelike Infinity, J. Math. Phys. 19 (1978) 549-554.

[9] R. Beig \& B.G. Schmidt: Einstein's Equations Near Spatial Infinity, Commun. Math. Phys. 87 (1982) 65-80. 
[10] A. Ashtekar \& J.D. Romano: Spatial Infinity as a Boundary of Space-Time, Class. Quantum Grav. 9 (1992) 1069-1100.

[11] S.M. Perng: On Conserved Quantities at Spatial Infinity, J. Math. Phys. 40 (1999) 1923-1950.

[12] C.W. Misner, K.S. Thorne \& J.A. Wheeler: Gravitation, Freeman \& Co., San Francisco 1973.

[13] M.W. Hirsch: Differential Topology, Springer, New York 1997.

[14] M. Forger \& H. Römer: Currents and the Energy-Momentum Tensor in Classical Field Theory: A Fresh Look at an Old Problem, Ann. Phys. 309 (2004) 306-389. 